\title{
The Nature of Gravitational Collapse
}

\section{Conrad Ranzan}

DSSU Research, Niagara Falls, Ontario, Canada

\section{Email address:}

ranzan@cellularuniverse.org

\section{To cite this article:}

Conrad Ranzan. The Nature of Gravitational Collapse. American Journal of Astronomy and Astrophysics. Vol. 4, No. 2, 2016, pp. 15-33. doi: 10.11648/j.ajaa.20160402.11

Received: May 11, 2016; Accepted: May 21, 2016; Published: June 4, 2016

\begin{abstract}
The presentation exploits several recent advances in understanding the nature of the Universe and its space medium. They include: (1) the DSSU theory of gravity, recently validated by successfully predicting observed patterns of galaxy clustering; (2) the new velocity-differential mechanism, a non-Doppler spectral shift; (3) the remarkably simplifying concept of particles, based on the photon, championed by physicist J. G. Williamson; (4) the unique subquantum medium founded in DSSU theory and the manner in which it conducts photons. Based on these concepts, which are essentially natural processes, it is clearly shown, with the aid of 15 figures, how the photon, the Universe's fundamental energy particle, is causatively linked to gravity and how it plays a major role in gravitational collapse. The main focus is on the nature of end-stage collapse and the processes that maintain a stable state; the detailed discussion includes the calculations of the mass and radius of the final collapsed structure - the Superneutron Star.
\end{abstract}

Keywords: Gravity, Gravitational Collapse, Space Medium, Aether, Black Hole, Neutron Density, Neutron Star, Superneutron Star, Aether Deprivation, DSSU Theory

\section{Background}

"Einstein believed that gravity and electromagnetism were 'all ye know on earth, and all ye need to know.' He considered them to be the fundamental forces of nature and thought that a theory which unified them could explain all the phenomena of nature. He even wrote a paper arguing that the force of gravity might be responsible for holding the atomic nucleus together." $-\mathrm{S}$. Glashow, Interactions

\subsection{Background to Understanding Gravitational Collapse}

Every mass body has an associated theoretical critical size calculable solely from its mass and energy content. If a body were to undergo contraction to its critical size, if we imagine the body being compressed to its critical size, then its gravity will effectively prevent any escape from the surface. Not even light would be able to escape, making it essentially invisible to outside observers. The surface that defines the critical size is called an event horizon. If the body has no angular momentum the event horizon takes the shape of a sphere and its radius is referred to as the Schwarzschild radius. Every star, every mass object, can be thought of as having a personalized Schwarzschild size defined by this radius.

In 1939 Einstein (1879-1955) published a paper in which he showed, using arguments based on special relativity, that matter could not be so condensed that the Schwarzschild radius would fall outside the gravitating body and thus become a reality [1]. In other words, a mass could never collapse to the extent that it would actually end up somewhere in the interior of its Schwarzschild sphere. Some mechanism, as yet undiscovered, would prevent such an occurrence.

Meanwhile, in the very same year 1939, J. Robert Oppenheimer (1904-1967) argued that when sufficient mass becomes concentrated it will collapse into its Schwarzschild radius, and it will do so because matter losses its stability to resist its own self gravitation. "When all thermonuclear sources of energy are exhausted a sufficiently heavy star will collapse." Unless the star is able to significantly reduce its mass content "to the order of that of the sun, this contraction will continue indefinitely." [2] In another paper, he had concluded that no law of physics was likely to intervene and stop at least some stars from collapsing through their Schwarzschild radius to become what were, decades later, called black holes. 
The influential Sir Arthur Eddington shared Einstein's view, believing that some unknown mechanism prevents the continuing collapse of matter [3]. In Eddington's words, "I think that there should be a law of nature to prevent the star from behaving in this absurd way." Eddington had wanted a law of nature forbidding such nonsense. He did not get one. But he had also mentioned 'various accidents' that might intervene, presumably referring to explosions that would disrupt the star before it had a chance to implode [4]. That law was not found until the first decade of the $21^{\text {st }}$ century.

Over the years the consensus view developed that Einstein was wrong - wrong in placing a reasonable restriction on the nature of gravitational collapse; and that Oppenheimer was right — right in his unrestrained formulation of the collapse. It turns out that Einstein had it right after all. And Oppenheimer had it wrong, wrong on two counts: on the claim or assumption that density increase has no limits and on the external location of the Schwarzschild boundary (leaving a region of empty space within).

Oppenheimer, however, was not entirely off the mark. He was right in the sense that gravitational collapse does occur. There really is no force to prevent it. But the nature of the end-stage collapse eluded all investigations during the past century. The actual mechanism of collapse is unlike anything Oppenheimer, his colleagues, or anyone else, might have imagined.

\subsection{Background to Understanding the Link Between the Photon and Gravity}

Coming back to Einstein, the familiar story is that this great man spent the last 30-plus years of his life searching for a workable unified field theory. In plain English, he was probing for the fundamental connection between the familiar photon - the electromagnetic wave-like particle of light - and the familiar gravity effect.

During his years at the Institute for Advanced Studies until his retirement in 1945 and at his Mercer Street residence until his death a decade later, he was involved in a "persistent search for a unified field theory. He never found it. He worked on a variety of solutions; each seemed to offer hope; each had eventually to be discarded."'[5]

The photon-and-gravity connection is the key to understanding gravity - particularly in extreme gravitational states of matter.

\subsection{Background to Resolving the Mystery of Gravitational Collapse}

A number of important developments over recent years have made it possible, for the first time, to understand the long-hidden nature of gravitational collapse. First and foremost, there is the discovery and unravelling of the nature of the universal space medium [6] [7]. This has made possible a multi-level understanding of gravity. Another development is the physical nature of length contraction; this is a phenomenon that must be incorporated into the collapse mechanism. There is also the remarkable particle theory of physicist John Graeme Williamson. Then there is what is probably the most significant astrophysics discovery of the last few decades and is currently driving a revolution in cosmology. I am referring to the velocity-differential mechanism of spectral shift (intrinsic redshift and blueshift).

One more development, the discovery and incorporation of a process unlike anything the physics community has ever encountered. It will be detailed as the resolution unfolds within the following pages; but be assured it is a provable feature of the natural world. It is the use of this feature that has definitively explained the cause of gravitation [8], the cause of galaxy rotation [9], the cellular structure of the universe [10] [11], the resolution of the Dark Matter mystery, and the resolution of the foremost structural anomaly in astronomy and astrophysics [12].

As a consequence of these advances, the following description of the mechanism of gravitational collapse is based on a sound foundation - including a proven process that drives our natural universe and makes it comprehensible like never before. What follows is a presentation of the real world, not a mathematical speculation, not an imagined abstraction; it is a presentation of the actual workings of the universe relating to gravity and gravitational collapse; it is not a mere model, but a self-consistent theory that works and explains why it works.

What follows is not a "what if" exercise.

It will be shown how the photon, the universe's fundamental energy particle, is causally linked to gravity and how it plays a key role in gravitational collapse.

\section{The Amazing Photon}

\subsection{The Fundamental Particle of Energy}

Light is simultaneously a wave and a particle; we therefore speak of light waves and also of light particles (called photons) with cyclic, or oscillating, behavior. The photon has electric properties and magnetic properties and so is referred to as an electromagnetic wave, or particle, or wavelike particle.

Although conceived many years ago, Clerk Maxwell's conceptualization of the particle of light still remains relevant in capturing what is known of the photon's physical nature:

"[W]hatever light is, at each point of space there is something going on, whether displacement or rotation, or something not yet imagined, but which is certainly of the nature of a vector or directed quantity, the direction of which is normal to the direction of the ray ... the magnitude of this vector remains always the same, but its direction rotates round the direction of the ray so as to complete a revolution in the periodic time of the wave...the direction and the angular velocity of this vector are perfectly known, though the physical nature of the vector and its absolute direction at a given instant are uncertain...This vector is always perpendicular to the direction of the ray, and rotates about it a known number of times in a second..." [13]

As an energy particle, the photon holds a special status. It functions as the most fundamental energy particle of the 
Universe. The amount of energy the photon carries is determined by its oscillating rate, its frequency.

The conventional representation, as a sinusoidal wave composed of electric and magnetic fields, is given in Fig. 1. The sketch shows two features that are highly relevant for the construction of a unified theory of particles (a unified theory which, in turn, is key to a natural theory of gravitational collapse). They are: (i) The electric and magnetic field vectors are always directed perpendicular to the direction of the photon's motion. (ii) The photon is a longitudinally extended entity - it has a wavelength.

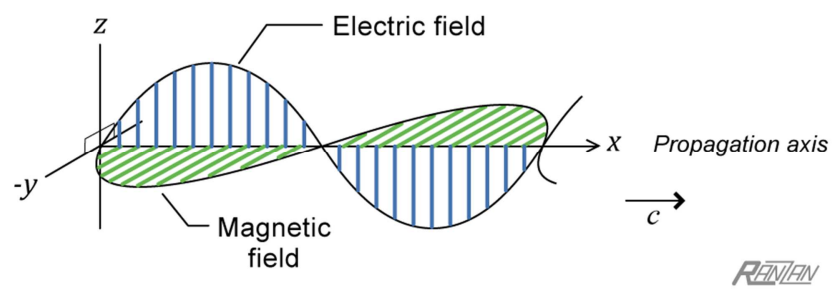

Fig. 1. The photon is an electromagnetic field (i.e., an excitation of the space medium) propagating as a wave. (It is shown here in the conventional manner with the electric force lines in the $x-z$ plane and the magnetic force lines in the $x$-y plane.) Photon's essential features: It possesses a wavelength (inversely proportional to the photon's energy); and its electric and magnetic field vectors are perpendicular to each other and to the direction of propagation. This longitudinal property and the vector configuration turn out to be important in explaining the nature of fundamental/subatomic particles and also in explaining profound aspects of end-stage collapse.

In addition to the ability of oscillating its electromagnetic field, the photon can rotate about the axis of its propagation - the photon can be induced to rotate in a manner independent of its intrinsic helicity (or characteristic spin). This allows the photon to be polarized in various ways.

Now let us examine the photon theory of particles in detail.

Consider the two photons shown in Fig. 2a. They are identical in that they represent the same quantum of energy, by virtue of having the same wavelength. They differ, however, by being phase shifted; they are 180 degrees, or one-half wavelength out of phase. Now, if the two photons are combined into a single structure, as in Fig. 2b, it is easy to see that the electric field vectors - being as they are, oppositely directed-will neutralize each other. So will the magnetic vectors. The linked photons effectively become invisible. In a world in which a single force-effect governs everything -where electromagnetics rules all interactions - the phase-shifted pair becomes a ghost particle. During the years between 1930 and 1962, the existence of such a particle, with no mass no charge and no trajectory track, was strongly suspected and was finally acknowledged. Enrico Fermi called it the neutrino [14]. Their elusiveness notwithstanding, neutrinos retain the energy of the constituent photons and retain the same speed of propagation. (This concept of the neutrino was inspired by the work of Eric Thompson, who seems to have been the first person to come up with the linked phase-shift idea. His research is published as Hydrocosmica (http://vixra.org/abs/1504.0023) and posted at: www.hydrocosmica.com)
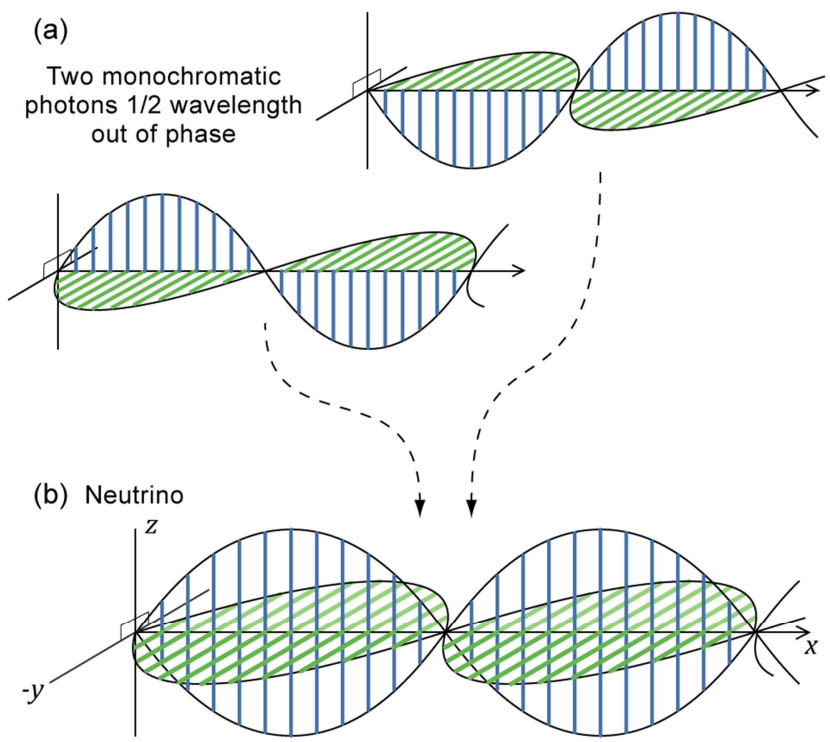

Two photons locked onto each other (their fields are intertwined)

Fig. 2. The neutrino is an energy particle consisting of two equal-wavelength photons that are $180^{\circ}$ out of phase. Their electric fields oppose each other and so do their magnetic fields; and consequently, the pair of embracing photons exhibits practically no external electromagnetic effect. They are said to be helically intertwined.

The neutrino's constituent photons retain not only their energy but also their spin (or angular momentum); and for the composite neutrino (and this includes all three types) the spin is always left-handed. This simplifies the explanation of the antineutrino. Given that the neutrino and its antiparticle are genuine mirror images of each other; the antineutrino is simply a right-handed spin particle.

For the next configuration, we make use of all three aforementioned photon properties - perpendicular fields, wavelength, and axial rotation. Let us rotate a single photon in the following manner: During the propagation of the photon along a distance of $1 / 2$ wavelength the photon rotates about the $x$-axis by $1 / 2$ turn $\left(180^{\circ}\right)$. This is in addition to its usual spin. While the peak electric vector moves from A to B, along the $x$-axis, the photon is making a half-turn rotation. The E-field wave-pattern will then look like Fig. 3b. (If there had been no rotation, the pattern would have looked like Fig. 3a.) If the photon continues this added rotation for another half cycle $(1 / 2$ wavelength), the $\mathrm{E}$ vectors for one entire wavelength will all be in the upward direction, as shown in Fig. 3c. In part (d) of the diagram, the magnetic vectors are included. Next, the pattern for one complete wavelength is drawn onto a strip of paper (Fig. 3e) and then reoriented so that the magnetic vectors are all pointing to the top of the page, while the electric vectors are directed outward from the page (Fig. 3f).

It is this circularly polarized photon, as represented by the paper-strip model, which we will now use to model the electron and the positron.

\subsection{Photon Confinement and the Property of Mass}

What the photon does not possess is the property of mass. And yet the photon is directly involved in the acquisition of 
the "mass" attribute. What must be understood is that mass, at this fundamental level (of the real physical world), is not a thing - it is a process. It will be explained shortly; but at the moment, the important point is that the photon acquires the property of mass the very instant it becomes spatially restricted. It all happens with photon confinement. So, what does that mean? ... It simply means that the photon is involved in a self-orbit arrangement. Our paper-strip model will serve to demonstrate how such confinement produces the two simplest autonomous mass particles.
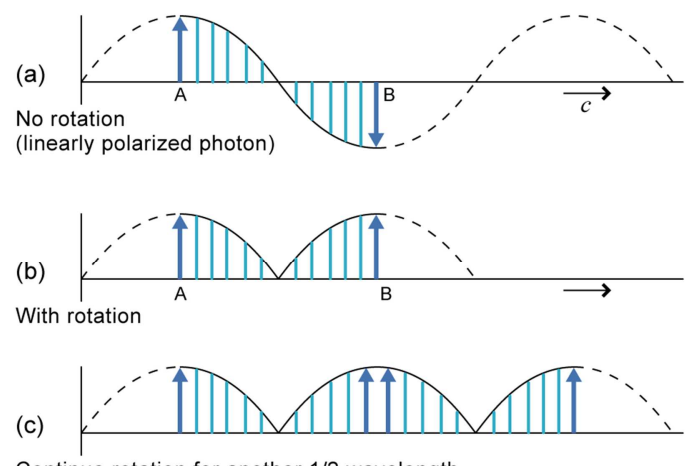

Continue rotation for another $1 / 2$ wavelength
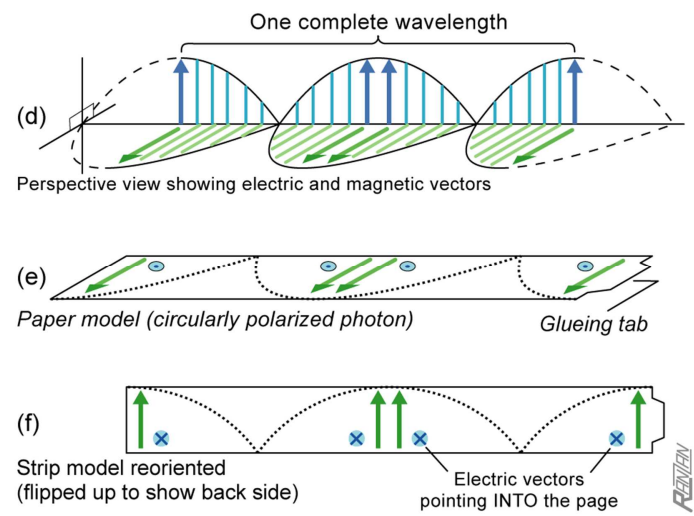

Fig. 3. Development of paper model of one wavelength of a circularly polarized photon. Start with the usual representation of an EM-wave with no additional rotation as shown in (a); this single wavelength is associated with a propagating photon of linear polarization. But if the peak electric-field vector (blue) pivots $180^{\circ}$ about the $x$-axis while it translates from position " $A$ " to " $B$," then the electric field (blue lines) will remain on the positive side of the axis as in Part (b). Apply another $180^{\circ}$ rotation to the peak vector during its progression along another half-wavelength distance; and an entire wavelength will have its electric field on the positive side of the $x-y$ plane as in (c). Part (d) shows both electric and magnetic field lines of the full-wavelength photon now in a state of circular polarization. In Part (e), the pattern is drawn onto a strip of paper; magnetic vectors (green) are drawn on both sides; the electric vectors are represented by blue highlighted dots to indicate the pointed end and by blue highlighted crosses to indicate the tail end. Part (f) is a reorientation obtained by simply flipping the paper strip so that the green arrows are pointing to the top of the page. The glueing tab points to the right (the same direction as the photon propagation).

The paper strip, representing a circularly polarized photon, is twisted into a helical shape as shown in Fig. $4 \mathrm{a}$ and Fig. 4b. Now note the orientation of the vectors; the magnetic force lines are all pointing upward; and the electric force lines are all pointing to the interior of the right-handed helix (but are all outwardly pointing for the left-handed helix). By then joining together the two ends of the strip model, confinement is complete - a single wavelength of radiation energy following a closed path (Fig. 4c). The result is a model of the electron, and its antiparticle, the positron. The upward-directed magnetic field models the magnetic dipole; the radial electric field vectors model the respective electric charges; and mass is modeled by the state of confinement.

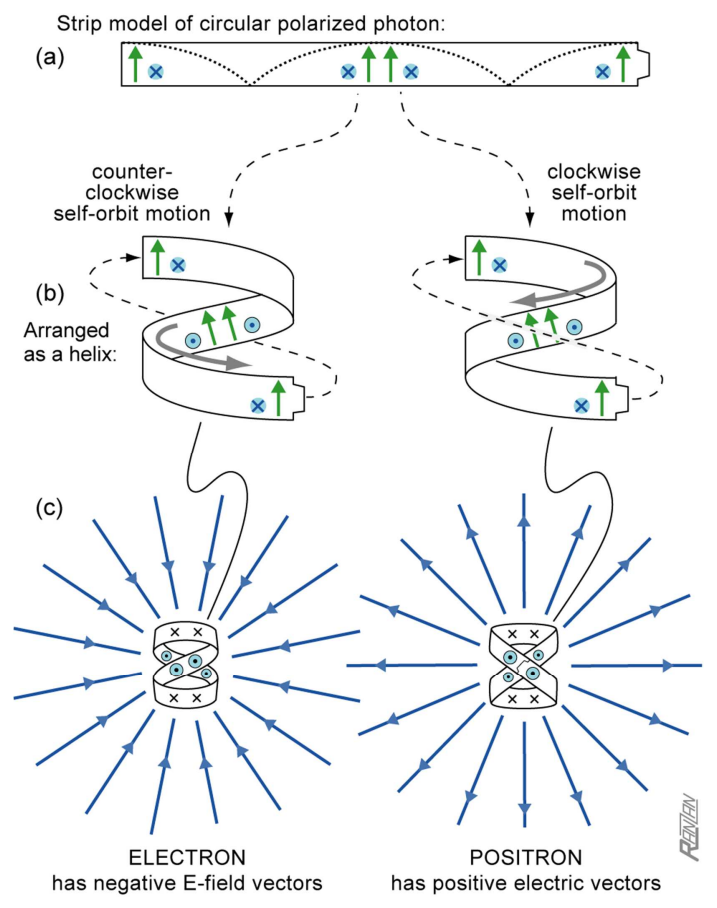

Fig. 4. The strip-model of the circularly polarized photon in (a) can be arranged in two ways as shown in (b). The helicity and rotation-sense of the confinement configuration determine the direction of the electric field vectors, which direction then determines the charge and the type of mass particle as in (c): The electron with its negative charge, the positron with its positive charge. (The symbol $\odot$ is for the point-end of a vector and $\otimes$ is for the tail-end.).

This model of the electron and positron is also consistent with what is understood about their formation and disintegration. The production of electron-positron pairs is known to occur when two high-energy photons collide under favorable conditions. And conversely, when an electron and a positron annihilate each other, two high-energy photons emerge from the collision.

The particle known as the muon has the same negative charge as the electron and the same spin (intrinsic angular momentum) but is considerably heavier than the electron. It may well be that the muon is a single wavelength photon twisted into a tight 4-loop helix. The pattern of magnetic and electric vectors would still be the same as for the electron. This argument may also be extended to the tauon - a super heavy version of the electron.

There is also a way to model the property of spin. Every elementary particle must possess, in addition to a characteristic mass, a certain spin (its intrinsic angular momentum). The looping and twisting motion of the confined photon is ideal for establishing a correspondence with a particle's spin property.

According to the new paradigm, all particles consist of 
electromagnetic loops (or loops of loops) - all particles are essentially confined photons. When these loops are complete, resonant, and harmonic they represent independent particles, such as the electron, muon, and tauon (and their antiparticle versions). However, when the electromagnetic loops are not complete configurations, then an interesting possibility arises. If a confined photon state is not sufficient in itself to complete a closed loop in space, then it may be possible to combine a number of such incomplete loops into a complete-and-stable combination. British Physicist J. G. Williamson identifies these incomplete loops with quarks. [15]

His remarkable insight is that the proton, the neutron, lambda, sigma, Xi, etc., - the baryons - are manifestations of a triple photon confinement; and the pion, the kaon, eta, etc., - the mesons - are manifestations of a twin photon confinement. And the electron, as has been demonstrated, is a confined single-wavelength circularly-polarized photon. [15]

What all this means is that mass consists entirely of confined (localized) photons. I say "entirely" because the gluon strong-force carriers are not required; they are replaced by the simple condition of loop closure or completeness (Fig. 5). By adopting such a principle of loop completeness, a force-mediating particle becomes entirely unnecessary.

(a)

Force-particle concept:

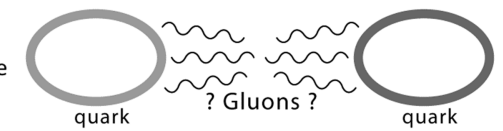

(b) Principle of oop completion:

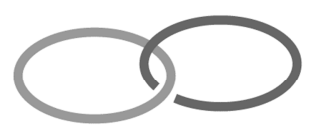

(c)

Strength of bond

increases with

(d) Triple-loop configurations:

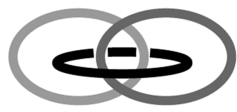

RENTIN

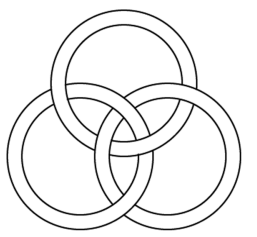

Fig. 5. Self-looping photon and the requirement of loop completion. The conventional concept of gluons as force carriers (a) can be replaced by the principle of loop completion (b). Any attempt to pull the quarks apart merely increases the strength of the bond (c), at least up to a certain point. Part (d) shows three-loop configurations (of which several other possible variations exist).

The strong nuclear force is replaced by the loop-closure principle. What about the so-called weak nuclear force? There are physicists who believe that the weak and electromagnetic forces might actually be two aspects of the same phenomenon. The legendary Julian S. Schwinger was known to have held this view [16]. In other words, the $W$ and $Z$ particles are merely photonic energy configurations.

Finally, we need not concern ourselves with the graviton the alleged force carrier for the gravitational force/effect. The graviton is a mathematical concoction. There is no such particle in the physical world. It is simply not needed - a conclusion the evidence will support as we continue with the photon's deep connection to gravity.

Here is our model so far: There is only a single fundamental force - and only one type of force particle. All particles are classified as (i) either free photons or combinations of free photons; or (ii) either confined photons or combinations of confined photons. ... Mass, generically described as a condensation of energy, takes on the specific meaning of photon self-looping confinement. All mass particles are composed of photons in the spirit of Williamson's bound-radiation theory of atomic particles. There appears to be no violation of physical laws.

The next step is to link the photon, the electromagnetic energy, to the medium that permeates all space.

\section{Linking the Photon to the Space Medium: Excitation}

Physicists are gradually coming to realize that there is a level of existence beneath the realm of reality of photons, quarks, electric-magnetic fields, and all quantum interactions. There is a growing awareness of a deep subquantum level, of some kind of space medium with which energy and mass particles interact in a very peculiar manner.

The idea of a space medium goes back to the $17^{\text {th }}$ century, to the theories of Rene Descartes (1596-1650). Philosophers and physicists of the $18^{\text {th }}$ and $19^{\text {th }}$ centuries made extensive use of the medium that pervades all space including the tiniest interstices of material objects - they called it the "aether" and speculated about its properties. The $20^{\text {th }}$ century saw a growing aversion to the concept of an aether; inexplicably, the evidence of aether detection, first in 1887 and most conclusively in 1925-1926, was completely ignored. Its existence was either ignored or simply denied and generations of students have been taught that light propagation requires no medium. The problem is, empty space is not nothingness. Some sort of medium is, and must be, present.

Evidently something is present. To say that a field, such as the electromagnetic field or the gravitational field, fills space is mathematically very convenient, but otherwise not at all satisfying. A "field" is little more than an equation specifying certain properties of a volume of space. But the question remains: What fills space?

The root of the problem in understanding the make-up of the space medium has been the failure to draw a distinction between what is measurable and a deeper substrate which is not measureable, at least not in the normal sense, not like measuring mass and energy. The experts misleadingly call the space medium the quantum foam - misleading because what is needed is a subquantum foam, a non-energy medium. They also misleadingly call it the vacuum of space - misleading because they claim their "vacuum" possesses energy, enormous amounts of energy, supposedly holding so much inexplicable energy that they have given it a special name, the 
vacuum energy problem. (There is also the problem of connecting the medium to the action of gravity, leading some scientists to explore the possibility "for a unified theory of the Standard Model and gravity in terms of an underlying, superfluid substrate of reality." [17] In other words, a substrate is needed to make gravity work.) They clearly need a concept below that of energy, but persist in seeking an explanation by hypothesizing new forms of energy.

No, the space medium (that underlies the quantum effects and energy itself and even gravity) is a subquantum medium - a non-energy substrate. In its static state, it possesses no energy, none whatsoever. This substrate is a non-mass-non-energy aether permeating all space. It is the essence of the Universe.

This aether plays a key role in the mechanism of gravitational collapse.

No mere speculation, the need for such a substrate was clearly recognized by Einstein, who stated:

"[W]e may say that according to the general theory of relativity space is endowed with physical qualities; in this sense, therefore, there exists an aether. According to the general theory of relativity space without aether is unthinkable; for in such space there not only would be no propagation of light, but also no possibility of existence for standards of space and time (measuring-rods and clocks), nor therefore any space-time intervals in the physical sense."

And he added a fundamentally important caveat: "But this aether may not be thought of as endowed with the quality characteristics of ponderable media ..." [18]

Furthermore, abundant supporting evidence for the existence of aether has accumulated over the many decades since the first detection in 1887 . There are now more than 8 well-documented experiments [19] [6].

The experiments do not specify the nature of the aether, beyond the fact that it exists and affects the propagation of photons. Naturally then, there are many different aether theories, many types of aether. So let me be clear on the distinctiveness of what is here proposed. The aether that we will use is the one that has been successfully exploited in a wide range of applications:

- in formulating the most plausible mechanism responsible for the cosmic redshift (described as a velocity-differential redshift);

- in providing the explanation, something long missing from astrophysics, of the ellipticity of comoving galaxies;

- in making possible a highly successful triple-process theory of gravity;

- in a powerful new resolution of Olbers' dark-night-sky paradox;

- in explaining the underlying mechanism that sustains cosmic large-scale structure.

We will use the aether upon which a complete-and-unified cosmology is based - the cosmology known as the Dynamic Steady State Universe (DSSU). It is this Worldview that has brought about a detailed understanding of the large-scale structural features of the Universe. Most notably, it has definitively unraveled the long-standing mystery surrounding the periodicity of the galaxy clusters associated with Abell 85 (itself a rich galaxy cluster).

In agreement with Einstein's view of aether, the DSSU medium is not a material substance; it possesses no mass and no energy. But unlike Einstein's aether, which was postulated as a continuum, DSSU aether consists of discrete units (non-material of course). Our aether is a true subquantum space medium.

In spite of its non-mass and non-energy nature, it does have several important properties. The properties that are of great relevance to the aether theory of gravity include the ability to expand (both axiomatically and when under tension) and contract (in the presence and vicinity of matter), and the axiomatic property of maintaining a constant spatial density (individual aether units per volume). In other words, DSSU aether serves as a dynamic medium.

The property, however, most relevant to the present discussion is the all-important ability to conduct quanta of electromagnetic radiation. This defines the aether as being luminiferous, making it the vital light-conducting medium of the universe. As Einstein had said (in his Leyden University lecture of 1920), without the presence of aether there "would be no propagation of light."

Einstein concluded that "the endeavour toward a unified view of the nature of forces leads to the hypothesis of an aether."'[20] How true indeed.

So, what is the nature of the "connection" between the photon and the space medium? What is the relationship between electromagnetic effects and the aether? Let us dispel the notion that the photon just pushes its way through aether as if it were something foreign and disconnected. We must understand that it is not something independent of aether. The photon is intimately linked to the space medium by being an excitation of the aether. Let me emphasis, the photon is the aether itself, a small portion thereof, in a state of excitation. And it is this excited state that is conducted by aether.

But what is the nature of the excitation? Historically, it has repeatedly been proposed that aether excitation involves tiny vortices, and swirls, that activate the lines of magnetic and electric forces. It was believed that vortices of aether constitute Faraday's familiar lines of force — vortices having left-handed and right-handed orientations, and arranged in diverging and converging patterns. This is almost certainly a valid aspect of the excitation. Still, one wonders, what is the difference between the magnetic and electric excitation states?

The problem is that the aether units, the essence fluctuators, the units directly involved in the excitation, are unimaginably small - it may be speculated that a single photon may involve trillions.

Here is what we can be quite certain of: The photon is a manifestation of the excitation of aether. The excitation is cyclical (rotational) and is associated with electric and magnetic "forces." And this cyclical nature is somehow responsible for propagation - the progression of the excitation "particle" and the speed of conduction. And it is also worth mentioning, energy resides in the excitation process, and not in the aether in its static state. 
There is one other aspect of aether excitation that must be explained. It turns out to be the key to understanding the mechanism of gravity and gravitational collapse.

\section{Crucial Aspect of Photon Propagation}

\subsection{A Most Unusual Mode of Conduction}

Light waves are not mechanical waves; they are not vibrations of the particles of a mechanical medium. True enough. But light waves may still be conducted by a "mechanical" medium! ("Mechanical" here does not imply mass or energy; it simply means that the medium, the aether, consists of discrete units.) Light may simply travel through the medium by a process of conduction - a conduction process that, in addition to whatever else is going on, consumes the "particles" of the medium. A photon, in DSSU theory, is a wave-like conduction-disturbance of aether. This "conduction," however, is unlike any other. The photon's mode of conduction is so strange, so unlike anything ever imagined, that any sort of analogy seems wholly inadequate.

The photon is conducted by aether in a manner that is destructive of aether.

This conduction process applies to all EM radiation and all entities that comprise atomic particles.

\subsection{Light Conduction Hypothesis}

The DSSU hypothesis of light conduction through aether-space requires that the photon propagates as an excitation process of aether, resulting in the destruction of aether units. Aether conducts the photonic packet of energy by momentary excitation followed by annihilation (total disappearance) of aether units/particles. See Fig. 6. The aspect of this process that is most important to our discussion is the accompanying flow of aether that tends to replace what was lost with the photon's passage.

Figure 6 makes no attempt to represent the electromagnetic mechanism; it makes no attempt to show the multitudinous vortices emanating from the heart of the excitation; nor does it attempt to represent the excitation. What the drawing does present is the unique consequence of excitation - the consequential annihilation of aether entities.

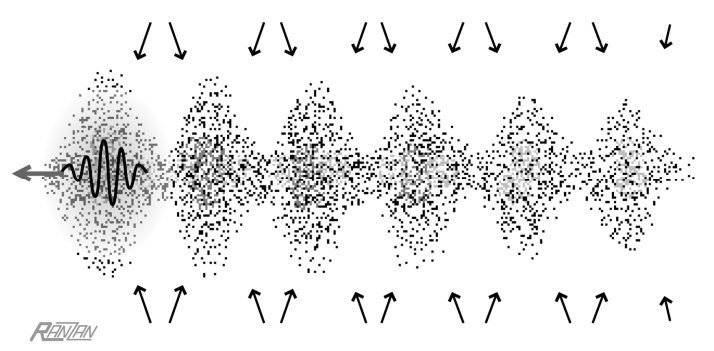

Fig. 6. Photon propagation. The photon, as an excitation of aether, is conducted by the aether in a manner that actually annihilates aether. In the process of conduction and excitation, aether units are destroyed. The dots represent a few of the vast numbers that are lost. The rows of arrows indicate a subtle flow of aether striving to replace what was lost with the photon's passage.
The photon's unique mode of conduction is extraordinary to a degree far beyond anything previously imagined. Its uniqueness is matched by its momentousness; for therein lies the heart of the emergent revolution in fundamental physics and cosmology. Conduction by aether excitation-annihilation defines the energy of radiation and, since mass is composed of photons; it also defines the energy of mass.

I cannot overemphasize the importance of this process. Not only is it the key to the success of the aether theory of contractile gravity, but it also makes possible a balanced universe. On a grand scale, the consumption of aether - by way of the conduction process - counters the production (expansion) of aether within cosmic Voids. The conduction process is what keeps the entire universe in its balanced state. Einstein considered the means by which the universe sustains a balance, as between the contraction tendency of gravity and the expansion tendency of the Lambda (his cosmological constant), to be immensely important. He viewed it as the secret of the universe [21].

\section{Consequences}

\subsection{Aether Flow}

All radiation particles, all energy particles, all mass particles, and all electromagnetic energy fields absorb/consume aether. Moreover, all contractile gravity fields consume aether, as will be explained in a moment.

The unique feature of mass particles is that the aether inflow is always in a radial pattern (although the shape of the pattern becomes distorted with accelerated motion). The simplest mass particle, the electron (or positron), is shown in Fig. 7a. Of absolute necessity for it to be a mass particle: there must be a photon; that photon must be confined; and the aether flow must be self-centric. Moving up the size scale, the water molecule, its protons and neutrons also configurations of confined photons, has a radial aether inflow (Fig. 7b). In fact, any natural aggregation of matter, such as a planet or a star as shown in Fig. 7c, produces a radial aether inflow.

The remarkable thing here is that the pattern is not only responsible for bestowing the property we call inertial mass, but it is also responsible for the effect we call gravitation. Without this pattern, there would be no attraction effect between uncharged bodies, no such thing as gravitational "pull." A simple thought experiment will confirm the amazing counterintuitive situation whereby an "infinite" slab of solid mass can have no gravitation! It would have a zero escape velocity. An infinite slab will produce a constant speed of aether flow regardless of the distance from the mass surface; and without a flow gradient, without a flow acceleration, gravitation is meaningless. Not even general relativity is able to predict gravitational acceleration for an infinite configuration. The radial pattern is essential. 


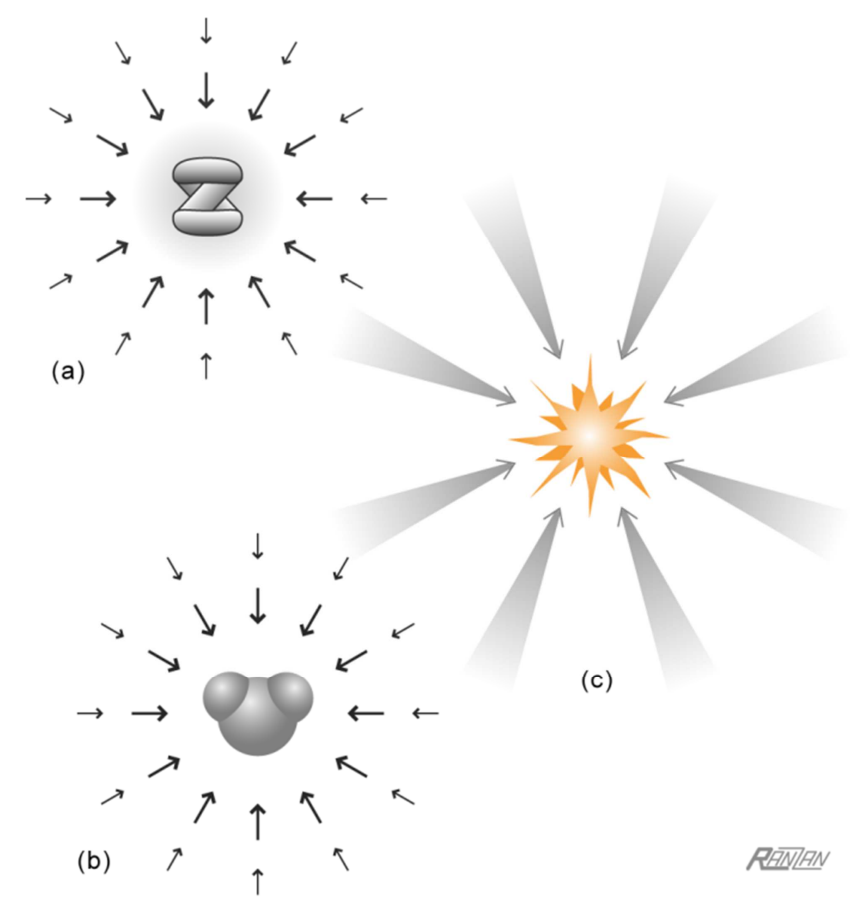

Fig. 7. Radial pattern of aether inflow for isolated "objects": (a) an elementary particle (electron or positron); (b) a simple molecule (water); (c) an astronomical body (star). It is this pattern that causes the flow to accelerate. It is this pattern that produces the inertial-mass effect. It is the acceleration of the medium within this pattern that produces the gravity effect.

There is more, not only does the pattern generate the gravity effect, but it also amplifies the effect. The radial inflow is important for two reasons. (i) It accelerates the flow of the aether, thereby making the flow region dynamic. (ii) Because of the acceleration, the aether becomes subjected to a form of self-pressure and responds by actually contracting and, thus, inducing a further increase in the flow acceleration.

Let me explain. The symmetrical pattern of inflowing aether tends to squeeze the aether units closer together, compressing the space medium. But recall, our aether always tends to maintain a constant count-density. The aether does this by self-dissipation, that is, a portion of the inflowing fluid literally disappears. As a result of the self-dissipation the inflow acceleration intensifies considerably —increasing the velocity much more than it otherwise would be.

The wonderful thing is that this effect is easy to measure and simple to calculate.

\subsection{Determining the Velocity of Aether Flow}

Let us quantify the inflow relating to large-scale bodies.

The test mass shown in Fig. 8 is resting on the surface of a planetary body. Although seemingly motionless, the object is "experiencing" acceleration. Two accelerations are involved: the platform on which the test mass rests is accelerating it upward; while the inflowing aether is accelerating it downward. The two are perfectly balanced, as evident by the lack of motion (with respect to the surface).

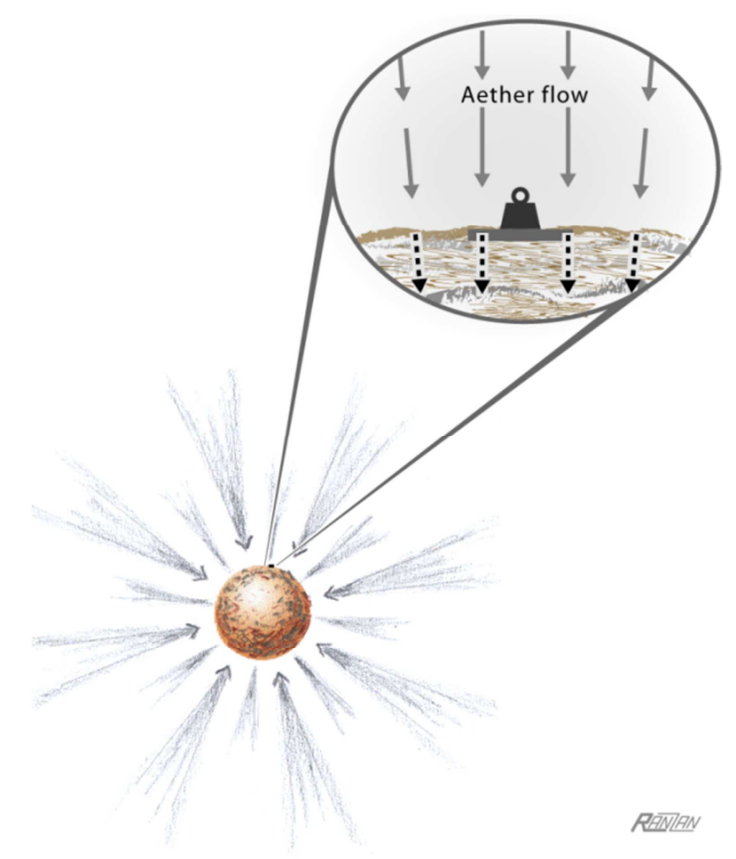

Fig. 8. Aether streams and accelerates towards and into the planet-sized mass. The surface test-mass "experiences" the inflow acceleration as a gravity effect, and "experiences" the inflow speed as a radial component of aether-referenced motion according to the expression in the text (eqn 6). The large body is assumed to be comoving with the cosmic background flow.

It is easy enough to prove that the platform on which the mass rests causes the test mass's upward accelerating: just remove all supports and watch the object as it freefalls. To convince yourself that the aether causes test mass's downward acceleration: Just imagine the removal of the aether inflow and picture the resulting test mass in a free-float state. Think of the removal of the radially inflowing aether, but the retention of the steady background aether flow. (Recall, matter cannot exist without the presence of aether, the matter-sustaining essence medium. In other words, aether must always be present. This requirement will be of critical importance in the discussion of the collapse mechanism.)

It is the downward acceleration - the radially inward inhomogeneous flow - of the aether that is of interest.

In order to express the flow mathematically, we will take advantage of the fact that the two accelerations, that of the object in freefall and that of the aether flow, are equal. We make use of the fact that the acceleration is directly proportional to the mass $M$ of the planetary body and inversely proportional to $R^{2}$ or $r^{2}$ (the square of its distance to the center of the planetary body). The equation looks like this:

$$
a=-(\text { constant }) \times \frac{M}{r^{2}} .
$$

The constant of proportionality is, of course, " $G$ " — whose value the Englishman Henry Cavendish, back in 1798, experimentally determined to be about $6.67 \times 10^{-11} \mathrm{~N} \mathrm{~m}^{2} / \mathrm{kg}$. For any location at, or above, the planet's surface, this acceleration expression describes a body in freefall as well as the aether inflow. 


$$
a=-G \frac{M}{r^{2}}, \text { where } \mathrm{r} \geq \mathrm{R} .
$$

Replace $a$ with its definition $d v / d t$ and apply the chain rule:

$$
\frac{d v}{d t}=\frac{d v}{d r} \frac{d r}{d t}=-\frac{G M}{r^{2}} .
$$

Then replace $d r / d t$ with its identity $v$, rearrange terms, integrate, and solved for the velocity:

$$
\begin{aligned}
& \int v d v=-\int \frac{G M}{r^{2}} d r, \\
& \frac{v^{2}}{2}=-\frac{G M}{-r}+C .
\end{aligned}
$$

Now, since the test mass (in Fig. 8) is stationary, its distance to the center of the planetary body is fixed, the velocity in the equation must be related to the aether. It must be related to the radial inflow of aether. Notice, there are two perspectives here: The aether is streaming downward past the test mass; but one could also say, the small mass is travelling upward through the aether. Both interpretations are embedded in the equation (and are made explicit in the next set of equations). In order to simplify the equation further, note that when the radial distance is extreme then obviously the aether inflow due specifically to mass $M$ must be virtually zero. (Keep in mind, we are assuming that the large body is comoving with the cosmic background flow; meaning that there is zero relative aether flow.) Mathematically, this means $C$ in the above equation equals zero. Thus,

$$
v^{2}=\frac{2 G M}{r} \text { and } v= \pm \sqrt{2 G M / r},
$$

where $G$ is the gravitational constant and $r$ is the radial distance (from the center of the mass $M$ ) to any position of interest, at the surface of $M$, or external to $M$. The positive solution expresses the "upward" motion of the test mass through the aether (in the positive radial direction). The negative solution represents the aether flow velocity (in the negative radial direction) streaming past the test mass.

The negative solution represents a spherically symmetrical inflow field - giving the speed of inflowing aether at any radial location specified by $r$.

In vector form:

$$
\vec{v}_{\text {flow }}=-\sqrt{2 G M / r} \times\left(\vec{r}_{\text {unit }}\right) .
$$

When a background aether flow is also present, as happens with objects within galaxies, the expression is

$$
\vec{v}_{\text {net flow }}=-\sqrt{2 G M / r} \times\left(\vec{r}_{\text {unit }}\right)+\left(\vec{v}_{\text {background }}\right) .
$$

A more detailed analysis of aether flow, in which a second gravitational constant " $\alpha$ " is included, is available in the works of physicist Reginald T. Cahill [22]. For our exploration of the collapsed state we only need to use eqn (6).

What about the flow occurring within the interior of a gravitating body? If we wish to know the inflow speed at some interior radial position $r$, then only the mass that is present inside the sphere of radius $r$ will be included in the equation. We denote the mass as $M_{\text {interior }}$ and express the magnitude of the velocity as

$$
v_{\text {inflow }}=\sqrt{\frac{2 G\left(M_{\text {interior }}\right)}{r}} .
$$

This is the equation we will be using in our exploration of collapsed stars of neutron density. For those ultimately dense bodies, our attention will be strictly focused on the flow velocity at the surface and the interior.

But first, let's take a closer look at how the Schwarzschild concept plays a role in the nature of gravitational collapse.

\section{Gravitational Collapse}

\subsection{Collapse: The Schwarzschild Radius Perspective}

Every mass object, regardless of size, has an associated theoretical radius called a Schwarzschild radius. The name comes from the German astronomer Karl Schwarzschild, who in 1916 worked out the basic concept whereby a sufficient mass concentration could produce a spherical event horizon; he had used Einstein's gravity to produce the same results that the British amateur astronomer John Mitchell had accomplished in 1783 using Newtonian gravity. For any mass particle, object, or body, it is possible to calculate and assign a Schwarzschild (event-horizon) radius.

If a mass body were somehow compressed to the size of its "personalized" Schwarzschild sphere, that sphere would have an aether inflow speed of about $300,000 \mathrm{~km} / \mathrm{s}$ - aether would be racing onto the surface of the gravitating sphere at lightspeed, on its way into the interior. (The conventional way of defining such a sphere is to say it has an escape velocity of $300,000 \mathrm{~km} / \mathrm{s}$ at the Schwarzschild surface.)

Let us use our Sun as an example: If the entire mass of the Sun were concentrated into a sphere of radius of $2.95 \mathrm{~km}$ we would have a Schwarzschild Sun. In the process of thus shrinking the Sun, not only would the surface gravity increase tremendously, but also the surface inflow would increase from its usual $617 \mathrm{~km} / \mathrm{s}$ to a staggering inflow of about 300,000 $\mathrm{km} / \mathrm{s}$. Needless to say, the Sun's density would have to be enormous to an extreme - beyond even nuclear density, the highest known density. Granted, the Schwarzschild concept is a well-defined mathematical construct; but our interest is with its correspondence to actuality.

So, what happens when a stellar-mass body, equivalent to say 4 or 5 Solar masses, undergoes gravitational collapse? What happens when the normal conventional counterforces of thermal expansion and electromagnetic forces are overcome, and only the nuclear force remains to resist the collapse? Because gravity is a cumulative effect, it would, in the absence of some limiting mechanism, be capable of overpowering each in its turn, first the thermal pressure, then the electron repulsion, and finally the neutron barrier.

Theoretically, at the completion of the collapse process or 
processes, there are only three possible configurations: (i) The body will have collapsed to end at some size external to the Schwarzschild radius; or (ii), to end at a size coincident with the Schwarzschild radius; or (iii), to end at a size interior to this radius.

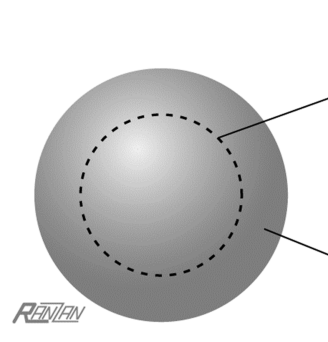

(a)

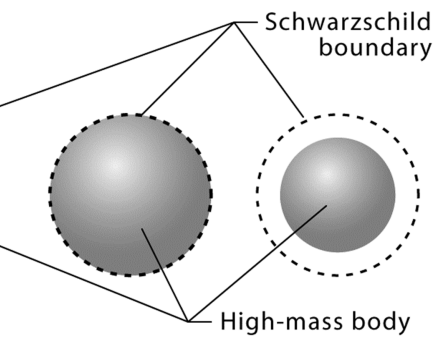

(b)

(c)
Fig. 9. Three possible collapse scenarios. Gravitational collapse of an inordinately massive body stops before reaching the Schwarzschild boundary, as shown in part (a). Collapse ends once the mass body has shrunk to the size of the Schwarzschild sphere, part (b). Collapse ends somewhere inside the Schwarzschild sphere, part (c).

The three possibilities are shown in Fig. 9. In each case, the mass of the body remains the same. And since the Schwarzschild radius depends entirely on the mass, it too remains the same. Consequently, the density must be different in each case; it is lowest for (a) and highest for (c).

With the first illustrated option, the dimensional size of the collapse halts somewhere outside the Schwarzschild boundary. The assumption here is that regardless of the total mass undergoing collapse the collapse will halt at some particular density, equal to or greater than neutron density. The problem here is that there is no known physics or hypothetical mechanism that could resist a continuation of the collapse. The nuclear degeneracy state cannot resist it; the proposed quantity of mass is too great. Furthermore, what happens when additional mass is added? This scenario fails - it merely represents an incomplete gravitational collapse.

With the second illustrated option, the collapse halts at the Schwarzschild boundary. The body attains a density that is the maximum at which mass-energy can exist. In order to comply with standard physics, its nominal density is the same as nuclear or neutron density. A simple mechanism prevents the body from increasing its mass content; in other words there is a mechanism by which additional mass undergoes total extinction. It is a truly remarkable object. It represents a stable gravitational collapsed state, yet has the potential for the total extinguishment of any addition of mass and energy. In short, it is capable of inducing the ultimate form of mass-energy collapse, as we will see momentarily.

With the final option, the collapse has proceeded through the Schwarzschild "surface" so that a high-mass-and-super-dense body resides inside the Schwarzschild sphere. A collapse that halts anywhere inside the Schwarzschild sphere, however, runs into serious problems, serious to the point of being a violation of the laws of physics. Such a collapse represents a clear violation of special relativity. It becomes obvious the moment one realizes that the velocity of the space medium inside the Schwarzschild boundary is greater than the lightspeed! Not only is it greater than the speed of light, but it increases even more as it flows toward the mass - and may be easily confirmed by solving eqn (9) using any value for the radius that is less than the Schwarzschild radius. This superluminal speed is a consequence of two facts: One is that the flow magnitude increases in inverse proportion to the square root of $r$, the smaller the radius the greater the speed. The other fact is that the speed of the space medium is not restricted by special relativity. But now imagine what would happen the instant the space medium were to reach its destination - the solid surface of the mass body - there would be a violation of the physical law that says nothing material can pass through space faster than lightspeed (and vice versa, the space medium cannot pass through matter faster than light). Thus, the scenario of Fig. 9c cannot represent a real object.

Now, as everyone knows, it has long been hypothesized that maybe the gravitational implosion collapses the entire material structure - in other words the surface itself ceases to exist. It is a radical attempt to overcome the special relativity violation - but utterly fails. A proper discussion of the paradoxes that burden such a hypothesis is beyond the scope of this article. Let me just briefly highlight a couple. By the definition of this type of a collapsed structure, all the mass energy located inside the event horizon is concentrated in one point (called a singularity). If this is so, then there can be no energy present between the singularity and its horizon (its Schwarzschild boundary). Remember, the singularity must necessarily absorb everything and every form of energy. But there is no getting away from the fact that the gravitational field is still needed - this field must still surround the singularity and extend out to the event horizon and beyond! But the gravitation field, undeniably, possesses energy and there is no way to explain its presence - therein lies the paradox.

The singularity serves to sweep the "material surface" (the component that blatantly violates Einstein's relativity) under a mathematical carpet -in an attempt to evade Einstein's objection of no mass surface inside the Schwarzschild sphere. The mass is deemed to be a singularity, a mathematical point with zero dimensions and no surface! With mathematical magic, the pros, like S. Hawking and R. Penrose, have made the surface disappear; they have taken a real representation and shrunk it down to an abstraction - an infinitely small point of infinite density. The special relativity problem has been solved; but at the price of a predicted infinite density for which there is absolutely no explanation! The singularity is admittedly a complete unknown.

Recapping the theoretical options (Table 1): Option (a) clearly represents an incomplete gravitational collapse; (b) is consistent with physical laws and consistent with the discussion in the previous sections; (c) violates physical laws and is plagued by paradoxes.

The self-evident conclusion is that in the real world an autonomous mass body never collapses through its Schwarzschild dimension. A reasonable approach leads to a theory in which, for single-object gravitational collapsed 
mass bodies, the event horizon coincides with the actual surface - the mass/energy surface.

Table 1. Three theoretical choices for gravitational collapse.

\begin{tabular}{|c|c|c|}
\hline \multicolumn{3}{|c|}{ Gravitational collapse } \\
\hline $\begin{array}{l}\text { Scenario (Defining } \\
\text { feature) }\end{array}$ & Comments & $\begin{array}{c}\text { Real } \\
\text { object? }\end{array}$ \\
\hline $\begin{array}{l}\text { (a) Collapse of an } \\
\text { inordinately massive } \\
\text { body stops before } \\
\text { reaching the } \\
\text { Schwarzschild boundary }\end{array}$ & $\begin{array}{l}\text { - This is an incomplete gravitational } \\
\text { collapse; full collapse requires } \\
\text { additional matter. }\end{array}$ & Yes \\
\hline $\begin{array}{l}\text { (b) Collapsed state } \\
\text { coincides with the } \\
\text { event-horizon boundary }\end{array}$ & $\begin{array}{l}\text { - A truly remarkable object. It } \\
\text { represents a gravitational collapsed } \\
\text { state with a potential for unlimited } \\
\text { mass-energy collapse (via unique } \\
\text { termination process). }\end{array}$ & Yes \\
\hline $\begin{array}{l}\text { (c) Collapse that halts } \\
\text { anywhere inside the } \\
\text { Schwarzschild sphere }\end{array}$ & $\begin{array}{l}\text { - Violates special relativity. } \\
\text { - Einstein had said ... some } \\
\text { unknown law of physics prevents a } \\
\text { body from collapsing through the } \\
\text { Schwarzschild boundary. }\end{array}$ & No \\
\hline
\end{tabular}

\subsection{The Superneutron Star}

No matter what the sequence of collapse may be - whether it is by way of nova or supernova events - once a sufficient quantity of matter has accumulated or a sufficient quantity of matter remains behind following any cataclysmic events the star may have undergone, then the end-state body will have the anatomical features shown in Fig. 10.

Let us start with a description of the aether flow. As we saw in connection with Fig. 8 earlier, the external inflow rate is proportional to $\sqrt{1 / r}$. The magnitude of the inflow increases with proximity to the body and reaches a maximum at the surface where it matches the speed of light. After passing through the surface (the event horizon), the aether flow decreases. During the course of the flow, as the aether is consumed by the neutron mass, the flow rate decreases approximately in direct proportion to the radius; and becomes zero at the very center. I say approximately because the neutronium density does not remain constant but varies, as we will see shortly.

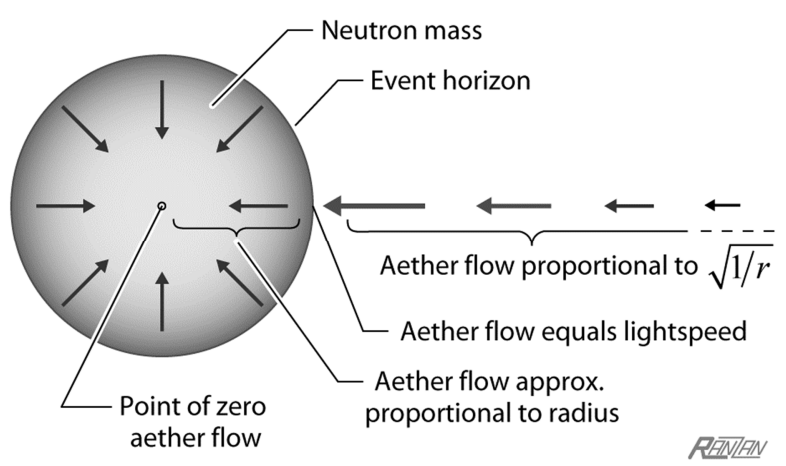

Fig. 10. Anatomy of a critical-state neutron star. Significantly, the surface coincides with the event horizon where the aether flow equals the speed of light. The external aether velocity is proportional to $(1 / r)^{1 / 2}$ (while comoving acceleration is proportional to $\left(1 / r^{2}\right)^{1 / 2}$. Because of a predictable variation in the density of the neutron mass, the internal flow speed is only approximately proportional to the radius.
Without question, the surface is the strangest feature. For the critical-state neutron star, the surface and the event horizon are one and the same. It has already been explained why there can be no empty space between interior matter and the event horizon - the simple fact being that nothing can travel through space faster than lightspeed. But at the neutron body's surface we have a situation where matter is actually moving, relative to the inflowing space medium, at lightspeed! How can this be? We have been taught that nothing, no material object or particle, can attain such a speed. It would require an infinite amount of energy! There is, however, this one place, one environ, where matter exists in a state, a steady state, of lightspeed motion. So, the next question is why is this state of motion, at the neutron-star surface, not a violation of physical law? ...

When a particle is forced to travel at lightspeed, the photon or photons that make up the particle are no longer able to complete their self-looping paths. They transform back into non-looping photons, back into linearly propagating EM energy. And these, of course, do travel at the requisite speed. Another way of describing the transition is to picture the extreme form of length contraction. When a particle attains lightspeed, it becomes, in a sense, a cross-section of its former self. In other words, if particles consist of self-orbiting photons, then a cross-section (a cross-section perpendicular to the direction of propagation) of the particle must manifest as a photon or some combination of photons, all travelling in the direction that the particle was heading. It seems this was understood even back in the 1920s. The Russian philosopher P. D. Ouspensky wrote, during this period,

"[An object] moving with the velocity of light would lose its third dimension. It would become a cross-section of itself. Lorentz himself affirmed that an electron actually disappeared when moving with the velocity of light.'[23]

In 1966, the eminent theorist John A. Wheeler wrote, “. .. in this extreme relativistic limit a particle of rest mass $m$ behaves ... in practically the same way as a photon."[24] The implication is that a neutron, travelling at the speed of light with respect to the aether, will take on the characteristics of a photon - a massless particle of pure energy.

But what was not understood back in the 1920 s, and for the rest of the century, was how such a situation could actually arise - how could matter be induced to travel at the speed of light? There are just two requirements: a mass of nominally neutron density and an event-horizon boundary.

Now, let us consider the surface from the perspective of energy. ... Ordinarily a particle at rest, say the neutron, has only mass energy and no kinetic energy. The neutron's confined photons normally follow tightly looping paths and the tightness of these paths define the particle's mass (the smaller the radius of the path, the greater the mass). As the speed of the neutron increases, the radius (with respect to aether) of the path increases, reflecting the fact that mass energy is being converted to kinetic energy (with respect to the aether frame of reference). Taking this to its logical conclusion, when the neutron is forced to travel at lightspeed, as is the case at the event horizon; its photons must necessarily follow the 
path of an infinite-radius loop. The formerly looping photons are now forced to travel in a linear direction - and such an entity has no mass. Remarkably the neutron's mass has faded to zero - and without mass there can be no mass energy and no kinetic energy. What remains is pure energy.

The surface of the critical-state neutron star consists of pure energy - an extremely thin layer of gamma photons. As shown in Fig. 11, the propagation axes of these photons are radially directed.

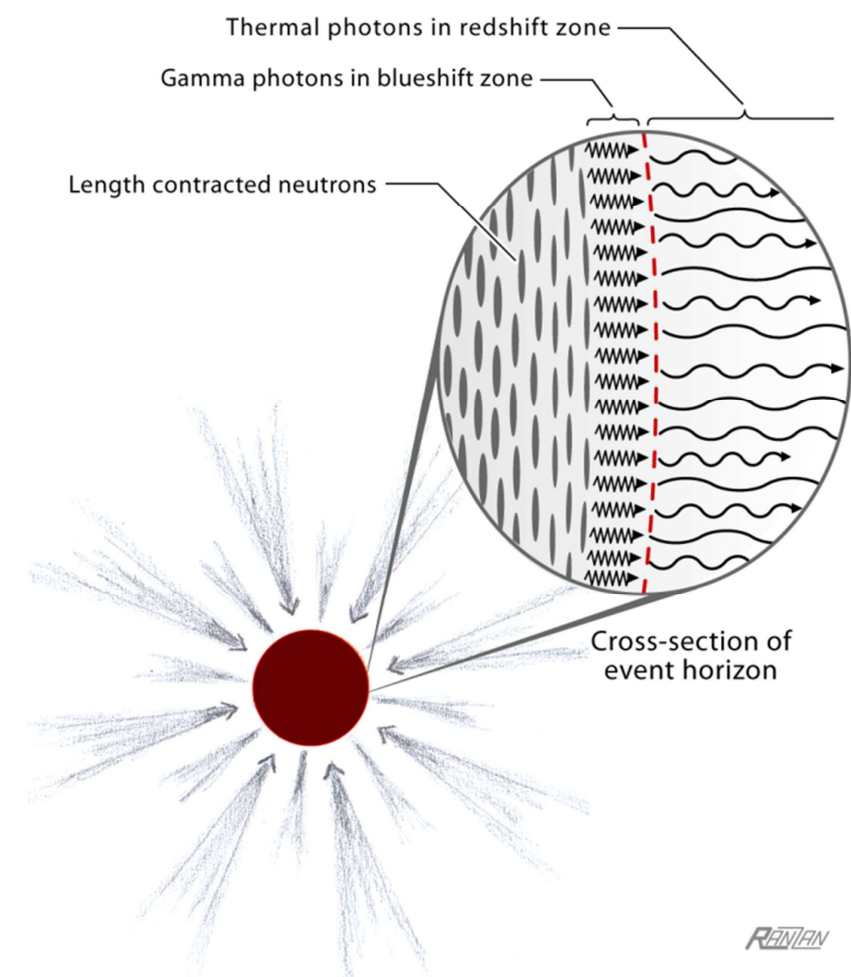

Fig. 11. The critical-state neutron star and its unique surface characteristics. The inner surface is where mass has been converted to pure photonic energy (gamma photons). These high-energy photons propagate outward at the body's inner surface; but because the aether is flowing inward with the same speed at which the photons are striving outward, the photons simply remain stationary at the surface, which also serves as the event horizon (red dashed line in the cross-section). On the external side of the boundary, we find "stationary" electromagnetic radiation undergoing a process of wavelength elongation - a clear example of velocity-differential redshift.

The profound implication of this: in the process of the formation of the event horizon, a quantity of mass undergoes a total conversion to energy. The $1.68 \times 10^{-27}-\mathrm{kg}$-mass of each neutron is converted completely to $939.6 \mathrm{MeV}$ of pure energy.

Adjacent to the surface, on the exterior side is a layer of trapped photons. Their wavelengths are radially oriented; and continually subjected to a velocity differential effect. In other words, these photons are being redshifted and most never manage to escape.

Turning our attention to the interior mass, we find it existing in its maximum allowable density state - mass there attains its ultimate density. Nominally, the density is the same as that of neutrons. But the actual density is most certainly higher. Let us examine the possible factors that may bring about an increase.
In general, there are three ways to increase density: (i) Change the atomic structure. (ii) Consider gravitationally induced pressure leading to further compression. (iii) Incorporate motion-through-aether length contraction.

Obviously there is no atomic structure available for altering - all we have to work with are densely-packed neutrons with a generally accepted density of $1.66 \times 10^{18} \mathrm{~kg} / \mathrm{m}^{3}$.

As for the second option, we see here a perfectly natural way to increase density; pressure does, in fact, increase from some minimum value near the surface to a maximum at the center of gravity. The problem is, at this stage in the investigation, we are not certain as to what quantitative factor to use. There is evidence to suggest that neutron density is very close (within a factor of about 2) to the maximum state-of-compression of gross matter. So, for now we will commit to the nominal density of neutronium.

There is, however, an additional factor to consider. It is the length contraction of the individual neutron particles, their change to an oblate shape. This shape flattening is due to the neutron particles' relative motion through aether. It is similar to the relativistic version but fundamentally different in that it is observer-independent and, therefore, is a physical effect. Physical length contraction is a very real phenomenon and is fully explained in the 2013 article "The Physical Nature of Length Contraction" where the contraction expression is independently derived without the use of the Lorentz transformation equations and without the use of Einstein's special relativity [25]. The phenomenon will, unquestionably, cause a physical increase in density.

It is this density-increasing process that must be taken into account when calculating the total mass and size of the critical-state neutron star. As we will see, it turns out to have a significant impact on both.

The result is that a critical-state neutron star must actually exist in a superneutron density state. Such a structure should properly be called a Superneutron Star $(\mathrm{SnS})$.

\subsection{Superneutron Star Mass and Radius}

Before working out the details for the $\mathrm{SnS}$, let us first calculate the size and mass of a basic neutron star (that is, without considering length contraction).

If we solve eqn (6) for the radius we get

$$
r=\frac{2 G M}{v_{\text {inflow }}^{2}} .
$$

At the event horizon, the speed of the aether inflow is $c$ - by definition. So, to find the radius of the event horizon we simply substitute $v_{\text {inflow }}^{2}=c^{2}$ and get

$$
R_{\text {critical }}=\frac{2 G M}{c^{2}}
$$

We do not yet know the total mass; but we do know the nominal density. Expressing the spherical (nonrotating) mass $M$ in terms of uniform density $\rho$, we have: 


$$
M=\frac{4 \pi}{3} R_{\text {critical }}^{3} \times \rho .
$$

When combined, eqns (11) and (12) give us

$$
R_{\text {critical }}=\frac{2 G\left(4 / 3 \pi R_{\text {critical }}^{3} \rho\right)}{c^{2}},
$$

which simplifies to

$$
R_{\text {critical }}=c / \sqrt{\frac{8}{3} \pi G \rho} .
$$

With the appropriate substitutions, we find

$$
\begin{aligned}
R_{\text {criticalN }} & =\frac{3.00 \times 10^{8} \mathrm{~m} / \mathrm{s}}{\sqrt{8 / 3 \pi\left(6.67 \times 10^{-11} \mathrm{Nm}^{2} / \mathrm{kg}^{2}\right)\left(1.66 \times 10^{18} \mathrm{~kg} / \mathrm{m}^{3}\right)}} \\
& =9.85 \mathrm{~km}
\end{aligned}
$$

for the radius of a basic neutron star.

We are now able to solve eqn (12) to find the corresponding total mass:

$$
\begin{aligned}
M_{\text {criticalN }} & =4 / 3 \pi R_{\text {critical }}^{3} \rho_{\text {neutron }} \\
& =4 / 3 \pi\left(9.85 \times 10^{3} \mathrm{~m}\right)^{3}\left(1.66 \times 10^{18} \mathrm{~kg} / \mathrm{m}^{3}\right) \\
& =6.64 \times 10^{30} \mathrm{~kg},
\end{aligned}
$$

which is equivalent to 3.32 Solar masses.

We now have the basic radius: 9.85 kilometers. It now needs to be "contracted" — not a simple matter. The problem is that the degree of length contraction depends on the aether speed - a speed which, as earlier pointed out, varies from maximum at one end of the radius to zero at the center-of-mass point.

To complicate the situation further, when the radius is reduced (shortened) it increases the inflow speed at the surface! The total mass cannot remain as just calculated!

So, how are we to incorporate the contraction of the neutrons?

First, we need to understand the concept of Euclidean space. It is unaffected by anything whatsoever. Euclidean space is the background "empty" space which is totally filled by the space medium which some call the vacuum and we call the aether. While the space medium goes about its business of expanding, contracting, flowing, and conducting EM excitations, the Euclidean space does absolutely nothing. It is, nevertheless, most useful. It provides a conceptual and mathematical framework in which to analyze the motions/dynamics of the aether and the matter it contains.

Second, we need the intrinsic contraction factor $\sqrt{1-\left(v_{\text {aether }}^{2} / c^{2}\right)}$.

Third, we need to understand that when one cubic meter of mass is subjected to length contraction only one of its three dimensions will be affected (assuming orthogonal aether flow). And this fact greatly simplifies the determination of the change in the density - it is simply the reciprocal of the contraction factor. So, while one dimension (say the height in Fig. 12a) decreases, the density increases according to the factor:

$$
1 / \sqrt{1-\left(v_{\text {aether }}^{2} / c^{2}\right)}
$$

Fourth, and because these calculations are done within a cubic meter of Euclidean Space, it means not only that the density will become greater but also that more mass will fit inside that same space.

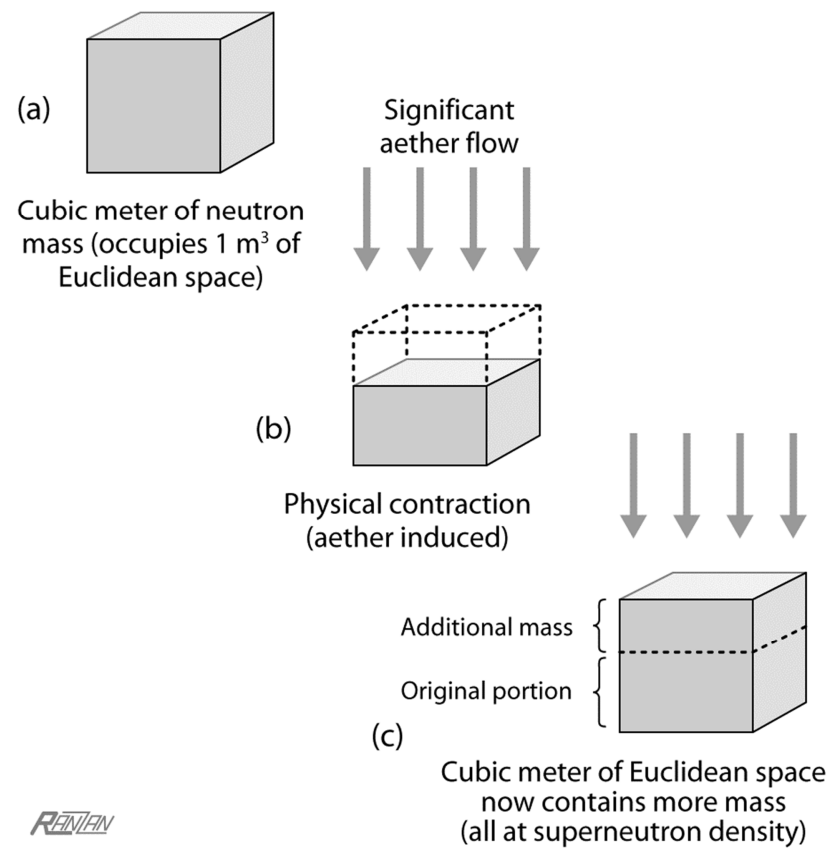

Fig. 12. How a cubic meter of Euclidean Space, filled with neutron mass, increases its total mass content. (a) One cubic meter of neutron mass, in the absence of aether flow, occupies a cubic meter of Euclidean Space. (b) Physical contraction caused by aether flow results in a new height that is $\left(1-v_{a}{ }^{2} / c^{2}\right)^{1 / 2}$ times the original. The result is an increase in the density by $a$ factor $1 /\left(1-v_{a}{ }^{2} / c^{2}\right)^{1 / 2}$. Part (c) shows the empty portion in (b) is now topped-up so that the cubic meter of Euclidean Space now contains more than the original mass and all of it at superneutron density.

Figure 12 illustrates the four points by which length contraction leads to a density increase. A neutron-mass cube with no motion with respect to the aether medium has no intrinsic length contraction (Fig. 12a). When there is aether flow (with significant speed) passing through the cube, as shown in Fig. 12b, then the height intrinsically contracts. Since only one of the cube's three dimensions changes, the resulting increase in density is easily calculated using the inverse of the contraction factor. The Euclidean-space cube, of course, never changes. So, if the neutron density has increased, then more of the same can be added to fill up the Euclidean one-meter cube (Fig. 12c). The essential point is that within a cubic meter of underlying Euclidean Space (within the neutron star) the density is: $\left(\rho_{\text {neutron }}\right) \times($ density factor $)$.

The density factor, which we will call gamma or $\gamma$, varies with the radial distance. What we need is a serviceable gamma 
function. Let us start with the reciprocal of the contraction factor and, thereby, express gamma as a function of the aether flow:

$$
\gamma(v)=1 / \sqrt{1-\left(v_{\text {aether }}^{2} / c^{2}\right)}
$$

The aether flow itself is a function of the two parameters $M$ and $r$, as per eqn (9). By combining eqns (9) and (16) we can express this density-factor function in terms of the radius and the mass inside that radius:

$$
\gamma(r, M)=1 / \sqrt{1-\frac{2 G\left(M_{\text {insde-r }}\right)}{r c^{2}}} .
$$

Now here is the problem: Say we want to find the density at the midpoint of the SnS's radius. We would need to know the total mass within that radius and to calculate this inner mass requires prior knowledge of the density. See the dilemma? To find the density one needs the mass; but to find the mass one first needs the density! In fact, one needs the densities of all the thin-layers that make up the inner mass structure. (Each thin layer requires a different valued density-factor.)

There is, however, nothing stopping us from using a step-by-step numerical method. Let us, then, apply the foregoing ideas and proceed to construct a $\mathrm{SnS}$ from scratch.

1. We start with an initial one-meter ball of neutron density. The contained mass is easily calculated and so is the magnitude of the aether inflow at the surface.

2 . The $v_{\text {inflow }}$ is then used to calculate the density factor in accordance with eqn (16). The value calculated for this factor will then be applied to the "thin" layer of mass to be added in the next step.

3. We surround our initial neutron ball with a "thin" layer of additional neutron mass. For calculating convenience and simplifying the discussion, we use thin layers that are 1-meter thick. We apply the density increase to the added-mass layer. Now at this point, as explained earlier, there is a choice: (i) We could allow our reference radius to contract along with the matter being contracted. Or (ii), we could keep the calculation radius at 2.0 meters by adding a little more neutron mass so that the total mass content is further increased (which now has a slightly higher density). In other words, in (ii), we add mass, let it contract, then add more, so that the added layer is ONE Euclidean meter thick.

It turns out that the latter approach is simpler and more intuitive. As we proceed to grow the mass incrementally, we will, therefore, use the background Euclidean space as a reference for the radial measure calculations.

4. Our construction now has a radius of 2 meters. The mass of the added layer is easily calculated using the density factor (calculated in step \#2), the base density, and the thin-shell volume. The total mass is simply the initial neutron ball plus the shell mass. And this is all we need, the radius and the total interior mass, to calculate (using only eqn (17)) the new density factor - applicable to the next layer to be added.

Although we are able to calculate each new density factor without evaluating the aether inflow, we do need to keep track of $v_{\text {inflow }}$ (just to ensure the structure's compliance with special relativity).

5. We add another 1-meter layer, determine its density and mass content, and add it to the previous mass total — and, of course, calculate the next density factor.

6. With each onion-layer addition, the radius is incremented, the mass is accumulated, the density factor is revised, and the inflow velocity is checked. The construction is complete only when $v_{\text {inflow }}$ attains the speed of light. See Fig. 13.

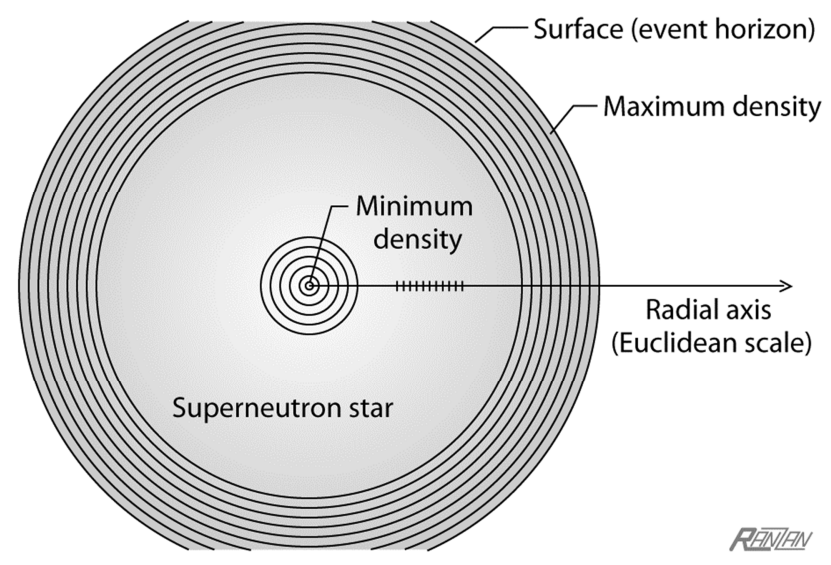

Fig. 13. Superneutron star divided into incremental mass layers (shown in cross-section) and used for a numerical calculation. The first incremental mass is a 1-meter-radius sphere. Additional mass increments are "thin" shells 1 meter in thickness. Minimum density occurs at the central "shell" (where it is considered to be of nominal neutron density). It increases to a maximum within the outermost layer. Density increase is due to length contraction caused by aether inflow, which is maximum at the surface and decreases to zero at the very center.

The mathematical construction of the $\mathrm{SnS}$ is quite straightforward, requiring only a basic iterative simulation. The flowchart of the computer program (and some additional details) that was used to obtain the following results is shown in the Appendix.

\subsection{Values for the Superneutron Star (Nonrotating)}

When the numerical simulation is performed - a simulation that considers the mass within a sequence of thin shells starting from an innermost "thin" shell and ending at the outermost shell where the aether inflow reaches a speed corresponding to lightspeed - the resulting values are:

- Final radius (for both structure and event horizon): 7.77 kilometers

- Total mass: 2.62 Solar masses, or $5.22 \times 10^{30}$ kilograms

- Final volume: $1.96 \times 10^{12}$ cubic meters

- Average density: $2.657 \times 10^{18} \mathrm{~kg} / \mathrm{m}^{3}$

- Average density (as a multiple of neutron density): 1.60

Now let us step back and examine what has happened. We began with a basic neutron star with a mass of 3.32 Suns and a radius of 9.85 kilometers. The mass within is sustained - its very existence is sustained - by the inflowing aether. The existence of this mass depends on a consumption rate (as calculated from the known surface area $12.2 \times 10^{8} \mathrm{~m}^{2}$ and the known surface-inflow speed $c$ ) of $36.6 \times 10^{16}$ cubic meters per 
second. This is all before length contraction was considered.

Then, after taking the density increase into account, we ended up with a SnS with mass 2.62 Suns and radius 7.77 kilometers. Something remarkable has happened! We have lost a significant amount of mass -0.70 Solar masses to be exact (the difference between 3.32 and 2.62 Suns).

Understand that the nature of the structure does not allow more mass to exist inside the now shrunken event horizon. More mass requires more aether, which in turn requires more aether to flow through the event horizon - and since the inflow has already reached the speed of light, a further increase is simply not possible. It is also not possible for the star to grow larger. This is because any growth in surface area (proportional to the radius squared) can't possibly keep up with the growth in mass volume (proportional to the radius cubed).

The question is What happened to the missing mass? How could two thirds of a Sun just vanish?!

Brace yourself, you are about to come face to face with a new law of physics.

\subsection{Process of Terminal Annihilation}

The most amazing aspect of our Superneutron Star: It has the ability to "collapse" any quantity of additional matter, any quantity whatsoever, without itself gaining mass! An understanding of this aspect requires an appreciation of the distinction between "gravitational collapse," on the one hand, and mass-energy collapse, on the other. Gravitational collapse should be thought of as merely the various mechanisms or stages by which matter becomes ever more concentrated. Gaseous atomic matter collapses and concentrates into liquid and solid states (planets); larger masses (small to medium-sized stars) collapse into a concentrated degenerate stars (known as a brown dwarfs); still larger ones, those with mass around 1.4 Solar masses, after burning up their nuclear fuel, concentrate into an electron degeneracy state (white dwarfs); and in a final stage of collapse-to-greater-concentration, when the mass exceeds the Chandrasekhar limit of about 1.4 Solar masses, matter transforms into the neutron degeneracy state (neuron stars). Matter and energy are always conserved.

Mass-energy collapse, however, is an entirely different kind of mechanism - a total mass-energy collapse in which nothing is conserved. It is a terminal collapse of mass and energy, a process under which nothing remains, nothing survives. And it can occur only at the core region, only at the point of zero flow (shown in Fig. 10), or in a vicinity encompassing that point of zero flow. It occurs when the $\mathrm{SnS}$ first forms and, thereafter, when additional matter falls onto the star.

Here's what happens when additional matter is absorbed: The event horizon expands outward momentarily; immediately thereafter, the central point-of-zero-flow expands into a sphere. The zero-flow point expands to become a zero-flow surface. Now, consider the neutronium inside this core region, inside this aether deprived zone. Without its sustaining supply of aether it cannot manifest itself, it undergoes terminal collapse
- it ceases to exist. Almost instantaneously, the hollow core collapses back to a point, sending incredibly powerful shock waves throughout the structure of the star. The collapse of the core, in an instant, restores the point of zero flow at the center of mass (until the next collision brings in more mass). The sequence of events occurs in extremely rapid succession; it all occurs in the time it takes for aether to flow from the surface (event horizon) to the center, a distance of less than $8 \mathrm{~km}$.

We can now explain what happened during the transition from a thought-experiment star (of basic neutron density) to a stable-state SnS (of superneutron density). See Fig. 14. During the transition, there was a $20 \%$ loss of mass (the 0.70 Solar mass). Here we invoke a new term for a new process. The mass lost in the transition is the result of aether deprivation.

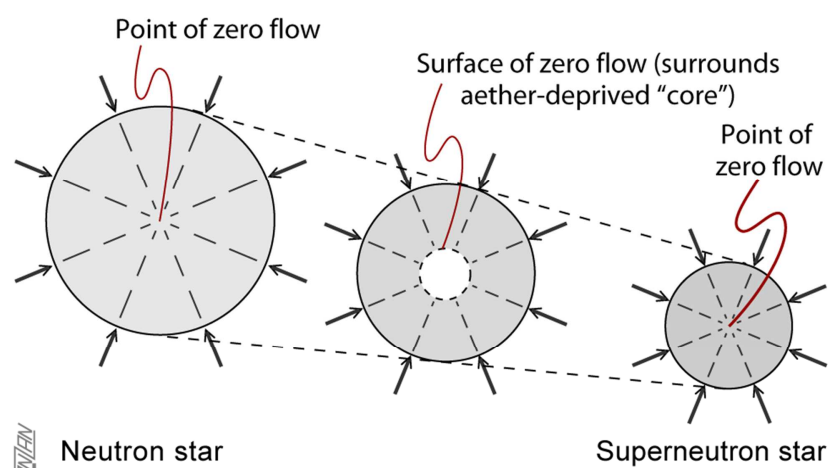

(a)

(b)

(c)

Fig. 14. Mass loss in the transition from a thought-experiment star (of basic neutron density) to a stable-state $\operatorname{SnS}$ (of superneutron density). (a) Unmodified neutron-density star of maximum allowable size: 3.27 Suns packed inside a $9.85-\mathrm{km}$ radius. (b) Because of physical length contraction of the contained particles, the body shrinks. The accompanying reduction in surface area reduces the quantity of aether reaching the core. But since matter cannot exist in the absence of aether, such an aether-deprived region must immediately collapse. (c) The body has reached its final stable size: 2.62 Suns packed inside a 7.77-km radius. The mass lost in the transition is a significant $20 \%$. The mass lost in the transition is the result of aether deprivation. (Not to scale).

Whenever the SnS gains energy, the gain feeds the aether deprivation process. There are two reasons why our SnS gains energy on a continuous basis. First, there is the CMBR. All the radiation that reaches the surface is retained; while no radiation ever escapes (as long as there is no rotation). Second, as shown earlier in Fig. 11, there is an ongoing process of blueshifting of subsurface photons. This represents a gain in energy. All such gains must be compensated for - at the core. Thus, mass loss through aether deprivation is an on-going process. Yet, the $\mathrm{SnS}$ itself neither gains nor losses any net mass.

Whenever the SnS absorbs mass, the absorption feeds the aether deprivation process. Quite naturally the SnS cannibalizes anything and everything in its neighborhood; everything from dust particles, through planets and stars, and all the way up to other SnSs. And again, there is compensating mass loss through the aether deprivation process — with no overall gain or loss to the $\mathrm{SnS}$ itself.

Because of this remarkable process, the concentration of matter is prevented from exceeding the superneutron density 
state, the formation of Oppenheimer's black holes becomes impossible, and the emergence of external Schwarzschild boundaries (in association with contiguous mass) is avoided. This is a new law of physics preventing collapse beyond the neutron-star state! It is called the law of matter extinction by aether deprivation.

\section{Concluding Discussion}

\subsection{Black Holes Versus Black Stars}

As we have seen, the $\operatorname{SnS}$ can absorb any quantity of additional matter and never grow larger, never become more massive, never collapse itself further. Essentially, it can never become a black hole. True enough, it possesses an event horizon, something it shares with black holes; but unlike the latter it can never sustain an empty region within its interior. Does this mean, then, that black holes do not exist? ... No it does not; it simply prohibits certain types of black holes. It categorically means that Oppenheimer's black holes and Penrose-and-Hawking's singularity black holes do not exist - except as mathematical concoctions. Einstein was right, "matter cannot be concentrated arbitrarily" [26] and the untrammeled implosion through the Schwarzschild radius was physically impossible [27]. Supermassive black holes, however, do exist and do so with almost no upper limit on their mass content. Although, supermassive black holes and our black stars are radically different animals, they do have one thing in common — both are bounded by an event horizon. Like everything else in the DSSU, they too have a simple and natural explanation.

So, while supermassive black holes do exist, they are not discussed in the present article. Our focus is on single star-like objects. The conventional belief is that the existence of an event horizon is the exclusive signature of a black hole. But this belief is wrong. In the context of extraordinarily massive stars, the existence of an event horizon is exclusively indicative of a SnS. In our real world, there are no small black holes concealing internal empty regions. There are no singularity black holes. There are, however, black stars with event horizons.

\subsection{How Physicists Were Led to the Belief in Basic Black Holes}

Physicists have long believed in collapsed structures in accordance with the following generally accepted definition: "A black hole is formed when a star collapses inward to cross its Schwarzschild radius."[28]. In terms of the choices available for the Schwarzschild boundary as presented in Fig. 9, they placed their faith in the third option. Once committed to this belief, they found themselves trapped. The nature of the matter located somewhere inside the Schwarzschild black-hole boundary was a veritable mystery. If it has a structure, then it would, as Einstein had warned, represent a violation of special relativity. Is this serious? Most certainly; it would mean the violation of the physical law that nothing can move faster than lightspeed! If, on the other hand, the contained matter has no structure and somehow exists as a zero-dimensional point, then it would represent a blatant disregard of Einstein's warning that matter cannot be arbitrarily concentrated -it would mean embracing a framework that is no longer in the realm of physics. Serious indeed.

One has to wonder why anyone would adopt such a strange and unnatural concept, and take on its formidable problems.

What was it then that led physicists to the belief in black holes as physical objects? ... In hindsight, two factors stand out: (i) The failure to understand the true nature of the event horizon, its nature as a quasi-physical boundary, its nature as the boundary for a SnS. (ii) The failure to understand what happens to additional matter passing through such boundary; and the related failure to appreciate that a universe capable of creating matter is also capable of extinguishing matter.

Most profoundly, though, what they failed to realize is that the law of conservation of mass and energy involves one conversion process that applies not to the collapsed gravitational system in isolation but, rather, to the entire gravity domain - a definable domain of which the $\mathrm{SnS}$ is the terminal component. In some parts of the domain (in the cosmic Voids) matter emerges, while in other parts (the SnSs) matter expires. Unrecognized was a balanced harmony of opposites acting in compliance with a broad interpretation of the conservation law.

\subsection{New Physics, New Understanding}

In 1931, Subrahmanyan Chandrasekhar (1910-1995) calculated that a non-rotating body of electron-degenerate matter above a certain limiting mass of about $1.4 \mathrm{M} \odot$ (now called the Chandrasekhar limit) would be unstable. He had found the limit at which the electron self-repulsion can no longer resist further gravitational collapse. White dwarfs, as these electron degenerate structures were called, could not exist as such above the 1.4-Solar-mass limit. His inference was opposed by many of his contemporaries, including Eddington and Lev Landau, who argued that some yet unknown mechanism would stop the collapse. Meanwhile, in 1932, the English physicist James Chadwick (1891-1974) discovered the neutron particle. It was soon realized that a higher density structure could be achieved with a dense packing of these neutrons, formed as they are from the pressure-induced union of electrons with protons. Because of the Pauli Exclusion Principle, a neutron degeneracy state would form a new stable barrier to further stellar collapse. Thus, a white dwarf slightly more massive than the Chandrasekhar limit will collapse into a neutron star. But then in 1939, Robert Oppenheimer and others predicted that a neutron star above approximately $3 M \odot$ (the TolmanOppenheimer-Volkoff limit) would collapse into a black hole. They concluded that no law of physics would prevent such a collapse [29].

But their conclusion was wrong. And people like Eddington, Landau and Einstein were right. It turns out there is a law of physics that prevents collapse beyond the neutron-star state!

The neutron degeneracy state is the final barrier in the 
evolutionary sequence of stellar collapse.

There is a law of physics - the law of matter extinction by aether deprivation - that prevents collapse beyond the neutron-star state! It is the physical law that prevents the concentration of matter beyond the superneutron density state; the law that prevents the formation of Oppenheimer's black holes; the law that prevents the formation of external Schwarzschild boundaries (in association with contiguous mass).

The new physical law of matter-extinction has profound implications: (1) A SnS can never grow in size or in mass content. A SnS can never become a supermassive black hole. A supermassive critical-state body (more properly called a "critical-state region") is an entirely different type of structure. The only shared characteristic is the presence of an event horizon. (2) The universe does not recycle! Matter is perpetually on a one-way journey of existence. The aether-deprivation mechanism within the $\mathrm{SnS}$ is the terminal phase of the journey. Existence ends in the heart of Superneutron Stars.

There is now also a new understanding of the physics underlying the event horizon - the SnS's quasi-solid surface. Notably, the $\mathrm{SnS}$ represents the discovery of the one-and-only energy conversion process by which mass undergoes a $100 \%$ conversion to photonic energy. It takes place only at the surface, only at the SnS's event horizon. This total conversion of mass to energy, of neutrons to gamma photons, is the natural consequence of the Williamson theory of particles and the DSSU aether theory of gravity (itself rooted in the photon's unique mode of propagation as described earlier). As a natural process of gamma particle formation, it becomes profoundly important for understanding and predicting the nature of rotating SnSs.

It has been shown, as promised, how the photon (as the particle of the one-and-only fundamental force of nature) is, by its interaction with aether, responsible for contractile gravity and, in extreme instances, for end-stage gravitational collapse.

Thus the endeavour toward a unified view of the nature of forces leads to the hypothesis of an aether.

- A. Einstein, 1922

\section{Appendix}

\section{A1. Glossary}

Aether: The subquantum medium that permeates all space. It is the nonmaterial essence of the Universe.

Aether deprivation: The termination process by which matter is extinguished. Matter does not and cannot exist in the absence of aether. When matter finds itself in a region of insufficient aether flow, a situation that can only arise at the bottom of a gravity well (sink) powerful enough to possess an event horizon, it ceases to exist.

Black hole: According to the conventional view, it is any gravitating object, or region, possessing an event horizon.

Black hole (singularity): A black hole for which all of its mass is concentrated at a single central point. It does not exist except as a mathematical object.

Black star: another term for superneutron star.

Critical-state neutron star: see Superneutron star.

Critical-state star: Any star which during the course of gravitational contraction has acquired an event horizon.

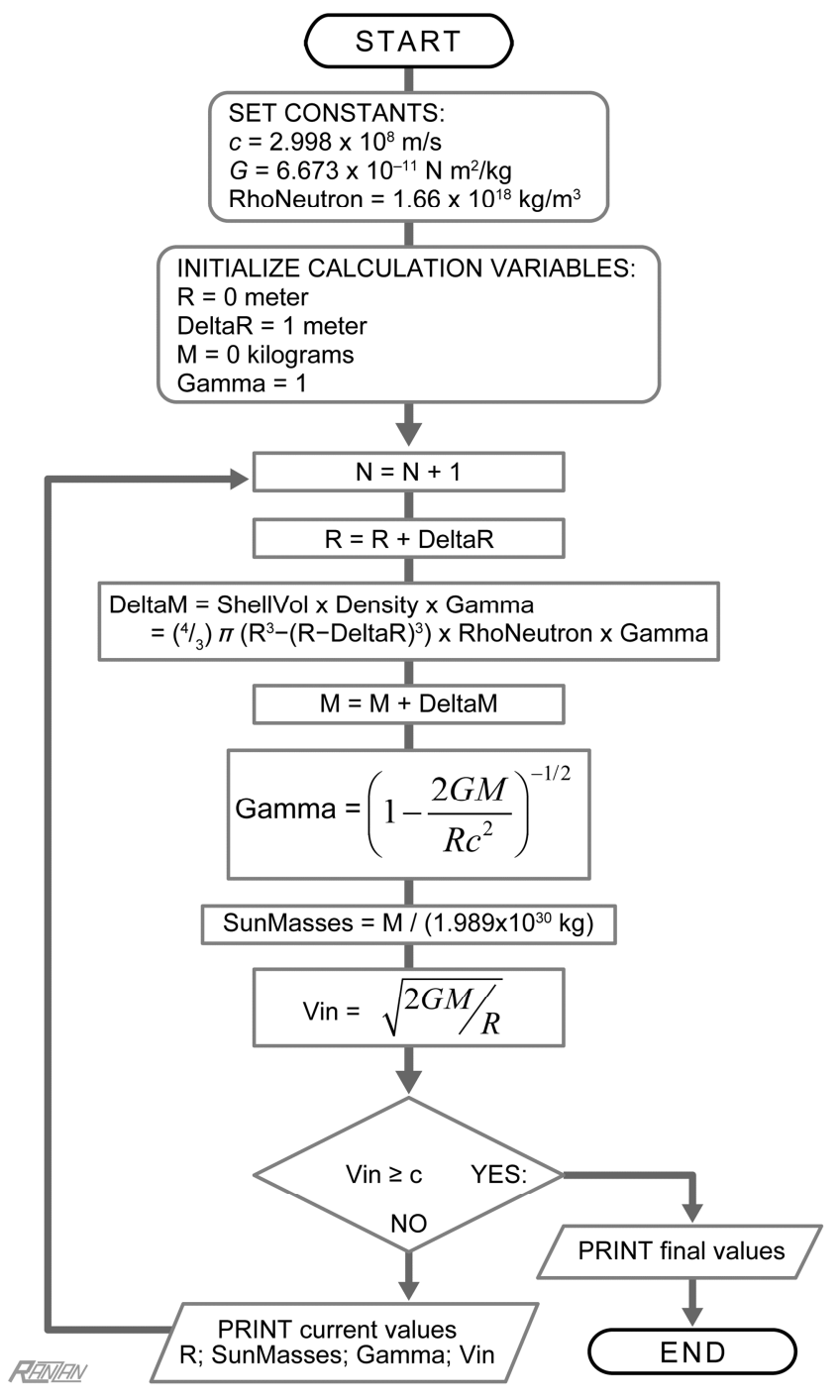

Fig. A1. Flowchart for numerical determination of characteristics of basic critical-state neutron star.

Table A1. Constants, variables, and expressions used in the superneutron-star numerical simulation.

\begin{tabular}{lll}
\hline Symbol & Value or Expression & Remarks \\
\hline & CONSTANTS & Speed of light \\
$\mathrm{c}$ & $c=2.998 \times 10^{8} \mathrm{~m} / \mathrm{s}$. & Gravitational constant $\left(\mathrm{N} \equiv \mathrm{kg} \mathrm{m} \mathrm{s} ~^{-2}\right)$ \\
$\mathrm{G}$ & $G=6.673 \times 10^{-11} \mathrm{~N} \mathrm{~m}^{2} / \mathrm{kg}^{2}$ & Basic neutron density $\left(\rho_{\text {neutron }}\right)$ \\
RhoNeutron & RhoNeutron $=1.66 \times 10^{18} \mathrm{~kg} / \mathrm{m}^{3}$ & \\
& Initial CALCULATION VARIABLES & \\
\hline
\end{tabular}




\begin{tabular}{|c|c|c|}
\hline Symbol & Value or Expression & Remarks \\
\hline R (initialized:) & $\mathrm{R}=0$ & This expression initializes the Radius \\
\hline DeltaR (constant) & DeltaR $=1$ meter & Radius increment. Constant during simulation. \\
\hline M (initialized:) & $\mathrm{M}=0$ kilograms & Initial spherical mass \\
\hline Gamma (initialized:) & Gamma $=1$ & Physical contraction factor \& density-factor function \\
\hline & For LOOPING PORTION & \\
\hline $\mathrm{N}$ & $\mathrm{N}=\mathrm{N}+1$ & Layer counter \\
\hline $\mathrm{R}$ & $\mathrm{R}=\mathrm{R}+$ DeltaR & Radius accumulator \\
\hline DeltaM & $\begin{array}{l}\text { DeltaM }=(\text { Vol of shell }) \times \text { density } \times \text { gamma } \\
\text { DeltaM }=(4 \pi / 3) \times\left(R^{3}-(R-\text { DeltaR })^{3}\right) \times \text { RhoNeutron } \times \text { Gamma }\end{array}$ & $\begin{array}{l}\text { Mass of thin shell being added. (The initial sphere is } \\
\text { considered to be the } 1^{\text {st }} \text { shell.) }\end{array}$ \\
\hline M & $\mathrm{M}=\mathrm{M}+$ DeltaM & Mass accumulator (mass inside radius $\mathrm{R}$ ) \\
\hline Gamma & Gamma $=\gamma=\frac{1}{\sqrt{1-\frac{2 G M}{R c^{2}}}}$ & $\begin{array}{l}\text { The density-factor function. } \\
\text { For the derivation see eqns (16) and (17) in Section } 6.3 .\end{array}$ \\
\hline SunMasses & SunMasses $=\mathrm{M} /\left(1.989 \times 10^{30} \mathrm{~kg}\right)$ & Mass accumulator converted to Solar masses (for printout) \\
\hline $\begin{array}{l}\text { SDF } \\
\text { (ShellDensityFactor) }\end{array}$ & $\mathrm{SDF}=($ DeltaM $) /($ ShellVol $\times$ RhoNeutron $)$ & $\begin{array}{l}\text { Shell density as a factor of neutron density. (Used as a } \\
\text { math check of } \gamma \text {.) }\end{array}$ \\
\hline \multirow[t]{2}{*}{ Vin } & $\operatorname{Vin}=\sqrt{2 G M / R}$ & Velocity of inflowing aether at $\mathrm{R}$ \\
\hline & $\begin{array}{l}\text { Vin }>\text { or }=c ? \ldots \ldots \ldots \ldots \ldots \ldots \ldots \ldots \ldots \ldots \ldots \ldots \ldots \text { If YES: } \\
\mathrm{R}=\text { a multiple of } n \text { meters? ................. If YES: } \\
\text { (" } \mathrm{n} \text { " depends on how many lines of printout are desired.) } \\
\text { Continue with next round through main loop. }\end{array}$ & $\begin{array}{l}\text { PRINT final values. END. (Test of velocity limit) } \\
\text { PRINT current values: } \\
\text { R; SunMasses; Gamma; SDF; Vin. }\end{array}$ \\
\hline
\end{tabular}

Note: The gamma factor always applies to the next layer (identified as $R+1$ ); $S D F$ applies to current layer (identified by $R$ ).

Dynamic Steady State Universe (DSSU): The cosmology theory founded on the view that the space-medium (a nonmaterial aether) is dynamic and that the medium expands and contracts regionally and equally resulting in a cosmic-scale cellularly-structured universe. It is a model based on the premise that all things are processes.

Event horizon: the boundary at which the speed of aether flow, with respect to the center of the gravitating structure or region, is equal to the speed of light. There are two types: (i) The quasi-solid event horizon is associated with superneutron stars. (ii) The free-space event horizon is associated with critical-state regions and supermassive black holes.

Matter extinction law: When matter is subjected to aether deprivation it ceases to exist.

Photon: The photon is a wavelike particle of radiation energy; it is the carrier of the electromagnetic force.

Special relativity speed rule: the rule that nothing can travel faster than $300,000 \mathrm{~km} / \mathrm{s}$ through space, or through aether.

Supermassive black holes: A structure delineated by an event horizon that surrounds an interior region of noncontiguous mass.

Superneutron star: A superneutron star may be thought of as a natural-type black hole; it has an event horizon but no empty region within. "Superneutron" refers to the density of the star's matter, which is greater than neutron/nuclear density because of length contraction of the constituent particles. The orientation of the length contraction coincides with the radial direction from the star's center.

Terminal-state star: a superneutron star that is actively absorbing/consuming additional matter. Terminal-state stars are the matter disposers of the universe - the ultimate mass-and-energy destroyers.

\section{A2. Simulation for Determination of Characteristics of the Superneutron Star}

The program flowchart, Fig. A1 below, gives the framework for calculating the total mass of a nonrotating superneutron star $(\mathrm{SnS})$. The assumed density is the same as what is conventionally assigned to neutrons, $1.66 \times 10^{18} \mathrm{~kg} / \mathrm{m}^{3}$. This is the value used before length contraction is applied. The program simulation adds a 1-meter-thick layer of neutron mass for each pass through the loop - thus simulating an incrementally "growing" neutron star.

The formation of an event horizon (when the calculated expression for aether-flow speed reaches lightspeed) signals the completion of the $\mathrm{SnS}$ and the end of the simulation.

The program parameters and constants are identified and detailed in Table A1.

The simulation considers the mass within a sequence of "thin" shells starting from an innermost "shell" (a 1-meter radius sphere) and ending at the outermost shell where the aether inflow reaches a speed corresponding to lightspeed.

The final values for the Superneutron Star are given in Table A2 along with corresponding values for the basic neutron star (for which no account was made for length contraction).

Table A2. Numerically calculated values for the Superneutron Star and a comparison with the "basic" neutron star.

\begin{tabular}{|c|c|c|}
\hline Property & $\begin{array}{l}\text { Without considering } \\
\text { length contraction }\end{array}$ & Superneutron Star \\
\hline Radius (event horizon): & 9850 meters & 7769 meters \\
\hline Mass content: & $\begin{array}{l}3.32 \text { Solar masses } \equiv \\
6.64 \times 10^{30} \text { kilograms }\end{array}$ & $\begin{array}{l}\text { 2.624 Solar masses } \\
\equiv 5.22 \times 10^{30} \text { kilograms }\end{array}$ \\
\hline Volume $\left(4 / 3 \pi R^{3}\right)$ : & $4.00 \times 10^{12} \mathrm{~m}^{3}$ & $1.964 \times 10^{12} \mathrm{~m}^{3}$ \\
\hline Average density: & $1.66 \times 10^{18} \mathrm{~kg} / \mathrm{m}^{3}$ & $2.657 \times 10^{18} \mathrm{~kg} / \mathrm{m}^{3}$ \\
\hline $\begin{array}{l}\text { Average density (as a } \\
\text { fraction of neutron): }\end{array}$ & 1.0 & 1.600 \\
\hline
\end{tabular}




\section{References}

[1] P. G. Bergmann, The Riddle of Gravitation (Dover Publications, Inc., New York, 1992) p131.

[2] J. R. Oppenheimer and H. Snyder, "On continued gravitational contraction," Physical Review 56 (5): 455-459 (1939). Bibcode: 1939PhRv...56..455O. doi: 10.1103/PhysRev.56.455.

[3] S. Detweiler, "Resource letter BH-1: Black holes," American Journal of Physics 49 (5): 394-400 (1981). Bibcode: 1981 AmJPh..49..394D. doi: 10.1119/1.12686.

[4] G. Greenstein, Frozen Star (Freundlich Books, New York, 1983) p209.

[5] R. W. Ronald, Einstein: The Life and Times (The World Publishing Co., New York, 1971) p535.

[6] C. Ranzan, "The History of the Aether Theory," Web-article posted at: www.CellularUniverse.org/AA3AetherHistory.htm (accessed 2016/3/15).

[7] R. T. Cahill, "Space and gravitation," Magister Botanicus, Vol. 2, pp. 13-22 (January 2004). Posted at: http://www.mountainman.com.au/process_physics/index_of_p apers.htm

[8] C. Ranzan, "The processes of gravitation -The cause and mechanism of gravitation," Journal of Modern Physics and Applications, Vol. 2014, article: 3 (2014). Posted at: www.cellularuniverse.org/G6GravityProcesses-abst\&.htm

[9] C. Ranzan, Guide to the Construction of the Natural Universe (DSSU Research, Niagara Falls, Canada, 2014) www.cellularuniverse.org/

[10] C. Ranzan, "The story of gravity and Lambda-How the theory of Heraclitus solved the dark matter mystery," Physics Essays Vol. 23, No. 1, pp75-87 (2010 Mar). Posted at: www.cellularuniverse.org/index.htm

[11] C. Ranzan, "The Dynamic Steady State Universe," Physics Essays Vol. 27, No. 2, pp. 286-315 (2014 June) (doi: http://dx.doi.org/10.4006/0836-1398-27.2.286) Posted at: www.cellularuniverse.org/index.htm

[12] C. Ranzan, "DSSU validated by redshift theory and structural evidence," Physics Essays, Vol. 28, No. 4, pp 455-473 (2015 Dec) (Doi: 10.4006/0836-1398-28.4.455). Posted at: www.cellularuniverse.org/index.htm

[13] J. C. Maxwell, A Treatise on Electricity and Magnetism, Vol. 2 (Oxford at the Clarendon Press, 1873) (Macmillan and Co., London) p 418.

[14] D. Griffiths, Introduction to Elementary Particles (John Wiley \& Sons, Inc., Canada, 1987) p 22-24.

[15] J. G. Williamson, "On the nature of the electron and other particles," Conference presentation paper (p1). The Cybernetics Society 40th Anniversary Annual Conference (2008). Posted at www.cybsoc.org/cybcon2008prog.htm (accessed 2012/10/24)

[16] S. Glashow, Interactions (Warner Books, New York, 1988) p122.

[17] Web-article:

http://www.simonsfoundation.org/quanta/20140624fluidtestshi ntatconcretequantumreality/ (accessed 2015-4-10).

[18] A. Einstein, Sidelights on Relativity, translated by: G. B. Jeffery and W. Perret, Methuen \& Co. London, (1922); republished unabridged and unaltered: Dover, New York, (1983). P23.

[19] C. Ranzan, "The aether experiments and the impact on cosmology." Web-article (2009) posted at: (www.cellularuniverse.org/AA1 Aether\&Cosmology.htm).

[20] A. Einstein, Sidelights on Relativity, translated by G. B. Jeffery and W. Perrett (Methuen \& Co. Ltd., 36 Essex Street, London, 1922) p5.

[21] L. Susskind, The Cosmic Landscape, String Theory and the Illusion of Intelligent Design (Back Bay Books; Little, Brown and Co.; New York; 2006) p 71

[22] Webpage posting R. T. Cahill's research, Index of Papers: $\mathrm{http}: / /$ www.mountainman.com.au/process_physics/index of $p$ apers.htm

[23] P. D. Ouspensky, 1931, A New Model of the Universe (1971 edition, Vintage Books, New York) p 360.

[24] J. A. Wheeler and E. F. Taylor, Spacetime Physics (W. H. Freeman and Co., San Francisco, 1966) p 121.

[25] C. Ranzan, "The physical nature of length contraction," Applied Physics Research Vol. 5, No. 1 (2013 Feb) (Doi: 10.5539/apr.v5n1p87). Posted
www.cellularuniverse.org/index.htm

[26] A. Einstein, "On a stationary system with spherical symmetry consisting of many gravitating masses," Annals of Mathematics Vol. 40 (1939) p 922.

[27] P. Davies, About Time, Einstein's Unfinished Revolution (Simon \& Schuster Paperbacks, New York, 1995) p 113.

[28] G. Greenstein, Frozen Star (Freundlich Books, New York, 1983) p169.

[29] J. R. Oppenheimer and G. M. Volkoff, "On massive neutron cores," Physical Review 55 (4): 374-381 (1939). Bibcode: 1939 Ph Rv...55..374O. Doi: 10.1103/PhysRev.55.374. 